九州大学学術情報リポジトリ

Kyushu University Institutional Repository

\title{
Diffraction-amalgamated grain boundary tracking for mapping 3D crystallographic orientation and strain fields during plastic deformation
}

Toda, Hiroyuki

Department of Mechanical Engineering, Kyushu University

Kamiko, Takanobu

Department of Mechanical Engineering, Toyohashi University of Technology

Tanabe, Yasuto

Department of Mechanical Engineering, Kyushu University

Kobayashi, Masakazu

Department of Mechanical Engineering, Toyohashi University of Technology

他

http://hdl. hand le. net/2324/1800891

出版情報: Acta Materialia. 107, pp.310-324, 2016-04-01. ASM International バージョン：

権利関係 : 
Diffraction-amalgamated grain boundary tracking for mapping 3D crystallographic orientation and strain fields during plastic deformation

Hiroyuki Toda ${ }^{\mathrm{a}}$, Takanobu Kamiko ${ }^{\mathrm{b}}$, Yasuto Tanabe ${ }^{\mathrm{a}}$, Masakazu Kobayashi ${ }^{\mathrm{b}}$, D.J. Leclere $^{\mathrm{b}}$, Kentaro Uesugi ${ }^{\mathrm{c}}$, Akihisa Takeuchi ${ }^{\mathrm{c}}$ and Kyosuke Hirayama ${ }^{\mathrm{a}}$

a Department of Mechanical Engineering, Kyushu University, 744, Motooka, Nishi ward, Fukuoka, FUKUOKA 819-0395, Japan (e-mail: toda@mech.kyushu-u.ac.jp, tel: +81-928023246, fax: +81-928020001)

${ }^{\mathrm{b}}$ Department of Mechanical Engineering, Toyohashi University of Technology, 1-1, Hibarigaoka, Tempaku, Toyohashi, Aichi 441-8580, Japan

${ }^{c}$ Japan Synchrotron Radiation Research Institute, 1-1-1, Kouto, Sayo cho, Sayo gun, Hyogo 679-5148 Japan

\section{Abstract}

By amalgamating the X-ray diffraction technique with the grain boundary tracking technique, a novel method, diffraction-amalgamated grain boundary tracking (DAGT), has been developed. DAGT is a non-destructive in-situ analysis technique for characterising bulk materials, which can be applied up to near the point of fracture. It provides information about local crystal orientations and detailed grain morphologies in three dimensions, together with high-density strain mapping inside grains. As it obtains the grain morphologies by utilising X-ray imaging instead of X-ray diffraction, which latter is typically vulnerable to plastic deformation, DAGT is a fairly robust technique for analysing plastically deforming materials. Texture evolution and localised deformation behaviours have here been successfully characterised in $\mathrm{Al}-\mathrm{Cu}$ alloys, during tensile deformation of $27 \%$ in applied strain. The characteristic rotation behaviours of grains were identified, and attributed to the effects of interaction with adjacent grains on the basis of the 3D local orientation and plastic strain distributions. It has also been revealed that 3D strain distribution in grains is highly heterogeneous, which is not explained by known mechanisms such as simple incompatibility with adjacent grains or strain percolation through soft grains. It has been clarified that groups consisting of a few adjacent grains may deform coordinately, especially in shear and lateral deformation, and the characteristic deformation pattern is thereby formed on a mesoscopic scale. 


\section{Introduction}

A comprehensive description of the structure of polycrystalline materials is the basis for understanding their physical and mechanical characteristics. Conventional scientific principles concerning polycrystalline materials have, however, been developed based on two-dimensional observations of surface or thin films. A series of surface microscopic observations provides a certain amount of information on time evolution behaviours of deformation, damage and fracture in polycrystalline materials. Such information, however, may be considered unrepresentative of the bulk of a given material, in the sense that surface-specific phenomena are often observed. For example, free-edge stress singularity predominates near the intersection of free surfaces and grain boundaries, which inevitably causes the premature decohesion of grain boundaries, and inhomogeneous strain distribution [1]. The existence of a free surface also lowers the level of stress triaxiality, and damage initiation and growth are thereby significantly retarded [2]. To investigate actual phenomena in the bulk of a polycrystalline material, a sectioned surface can be examined, for example, with the electron back-scattering diffraction technique [3]. This, however, is a fundamentally destructive and non-sequential mode of observation, and any results should therefore be interpreted with caution, due to the three-dimensional (hereinafter 3D) complexity of microstructural features in practical materials. It has been vital, therefore, for other procedures to be developed, in order to obtain accurate 3D in-situ representations of polycrystalline materials.

Recent studies employing the latest synchrotron X-ray sources with large fluxes have been exploited for the development of several advanced techniques, which have made non-destructive 3D observations possible. These techniques include 3D X-ray diffraction (3DXRD) $[4,5]$, diffraction contrast tomography [6], and differential-aperture X-ray microscopy [7]. 3DXRD, one of the first such advances, allows for state-of-the-art analysis of polycrystalline materials, and has enabled Schmidt et al. and Poulsen et al., for example, to reveal characteristic recrystallisation behaviours $[8,9]$. All these techniques, however, require $\mathrm{X}$-ray diffraction in order to map grain boundaries; and this means that, due to the resulting occurrence of intensity reduction, spot blurring and spot overlap during plastic deformation [10, 11], the techniques are more or less vulnerable to plastic deformation. Spot overlap is mainly attributable to the orientation spread of individual grains. As a result of considerable efforts having been made to eliminate the effects of such spot overlap, for example by extensively 
simulating spot overlaps as functions of unit-cell size and resolution, overlapping spots of the order of $10 \%$ in fractional area can be weeded out using the sophisticated algorithms developed by Sørensen et al. $[12,13]$. Nevertheless, it has still been reported that such orientation spread often prohibits indexing of embedded grains in materials deformed by more than $10 \%$ [13].

The present authors have proposed a tomography-based method, called grain boundary tracking (hereinafter GBT), for imaging 3D polycrystalline structure and local strain distribution [14]. The X-ray microtomography (hereinafter XMT) images are capable of revealing microstructures such as particles and grain boundaries, and enable the reconstruction of grain boundaries in polygonal form, by identifying grain boundary particles. Furthermore, by tracking the positions of grain boundary particles throughout a given deformation process, it is possible to track the particles back to their original positions. Based on these results, it is possible to accurately determine grain morphology and its change during deformation, and to perform high-density strain mapping. This type of technique has been successfully utilized to study the fundamental deformation mechanisms of polycrystalline materials [14, 15], and has revealed strong interaction among neighbouring grains, and subsequent damage initiation and growth. Its first application to tensile deformation of an aluminium alloy revealed highly heterogeneous deformation in individual grains [14], contrary to the conventional understanding, which deformation is attributable to the complex and heterogeneous structures of actual polycrystals. In order to produce a physical interpretation of the complex deformation, the GBT technique has been modified to measure the crystallographic orientation of grains, by adding an X-ray diffraction experiment in the preliminary studies $[16,17]$. Determining which grain has produced which diffraction spot has been a fundamental problem when interpreting XRD images. A polycrystalline aluminium alloy deformed up to a unidirectional strain of $10.4 \%$ [16], or moderate deformation, in the vicinity of a fatigue crack, was analysed in order to develop a combined experimental technique; and this new technique is termed diffraction-amalgamated grain boundary tracking (hereinafter DAGT).

In the present study, the DAGT technique was applied to larger tensile deformation of a polycrystalline aluminium alloy, after modifying image processing procedures. The origins of the abovementioned heterogeneous deformation behaviours were here investigated by analysing the effects of crystallographic orientation for demonstration purposes.

2. Diffraction-amalgamated grain boundary tracking 


\subsection{Grain boundary tracking}

The GBT technique enables accurate reconstruction of 3D grain morphologies during deformation, even close to fracture [14]. In the GBT technique, the deformation behaviour of a specimen is observed in situ using the XMT technique. In general, microstructural features, such as particles, secondary phases and pores, are observed together with internal damage such as micro-cracks and cracks. The basic idea behind GBT is that a large number of microstructural features, which are visible in practical structural alloys with the currently available XMT technique, can be exploited as internal strain/displacement markers. Typically, 8,000-70,000 particles and/or micro pores are observed per $1 \mathrm{~mm}^{3}$, within a field of view of $0.3-0.5 \mathrm{~mm}^{3}$, for a $1 \mu \mathrm{m}$ spatial resolution XMT setup, in the case of wrought aluminium alloys [18]. Grain boundaries are, however, invisible due to their nanoscopic width, which is orders of magnitude less than the spatial resolution of the projection-type XMT technique.

In-situ observation of deformation behaviour is interrupted before the final rupture occurs, in order to accurately delineate grain boundaries by wetting grain boundaries with gallium [19]. The gallium-enhanced grain boundary image is binarised to produce a topographic relief by employing distance-transform and watershed algorithms. Precise image registration is then performed for the XMT images captured before and after gallium application, to identify particles located along the gallium-enhanced grain boundaries; and 3D marker-based registration is performed to calculate necessary image shifts in all the translational and rotational directions. Each grain is then reconstructed as a polygonal mesh by connecting particles, located along the grain boundaries, as plane-triangle components. This triangulation of the surface of individual grains is repeated at each loading step by employing the microstructural tracking technique (hereinafter MTT) [20], enabling visualisation of the deformation behaviour of each polygonal grain by tracking all the grain boundary particles throughout deformation. Details of the MTT technique are available elsewhere [20].

High-density 3D strain mapping is also achieved, by focusing on the physical displacement of particles located both in the grain interior and along grain boundaries. The combination of 4D morphological information on grains and 4D strain mapping has enabled a direct understanding of fundamental deformation mechanisms in polycrystalline materials $[14,15,17]$. Further details of the GBT process are available elsewhere [14].

2.2 Crystallographic indexing of grains visualised with the GBT tehnique

A 'pencil-beam' X-ray diffraction technique is coupled with the GBT technique. 
Diffraction spots are obtained from all the grains during the DAGT experiment, and then back-projected to a sample position, in order to calculate the crystallographic orientation of all the grains visualised with the GBT technique.

\subsubsection{Pencil-beam XRD technique}

Figure 1 is a schematic representation of the setup used in the pencil-beam XRD experiment, as part of the DAGT technique. A wide-area detector for XRD measurement (replacing the high-resolution detector for XMT observation) is positioned at an appropriate distance for XRD data collection. Monochromatic radiation from a monochromator is collimated with a Fresnel zone plate (hereinafter FZP) or slit collimator, to form an X-ray pencil beam, in order to facilitate data analysis by limiting the number of diffraction spots obtained for each X-ray path. By rotating the sample through $180^{\circ}$, the pencil beam can scan in both the lateral and horizontal directions, thereby illuminating every location in the region of interest, from all possible directions with respect to the rotation axis of the sample, at a typical scanning step of $10 \mu \mathrm{m}$. Since data collection is made on the fly during sample stage rotation, diffracted beams are exhaustively acquired. Although collimation greatly reduces the number of diffraction spots related to a particular grain, there remain a number of diffraction spots retained from grains which the pencil beam simultaneously intersects.

In the crystallographic indexing of individual grains, extraneous spots, which either originate from other grains along the pencil beam, or are generated by the double diffraction phenomenon [21], must be eliminated. The number of such extraneous spots is dependent on the relationship between sample size and grain size. Given the typical specimen size for the high-resolution projection-type XMT technique $(1000 \mu \mathrm{m}$ in diameter), and the common grain size of industrially produced alloys ( $20 \mu \mathrm{m}$ in this estimation), a fiftyfold number of diffraction spots is observed, even if only single diffraction is assumed. It is therefore indispensable to introduce an approach for indexing multigrain diffraction data, as presented below (Section 2.2.2).

\subsubsection{Crystallographic indexing}

A robust procedure for screening diffracted beam spots has been developed, to exploit the morphological information provided by the GBT process. Spatially resolved GBT data allows us to correlate the location of the origin of each diffraction spot with the location of individual grains, utilising information both on the locations of grain boundaries and on the gravity centre of grains. The algorithm consists of two processes executed simultaneously to evaluate the likelihood that a given grain has generated a particular diffraction spot. 
The two processes evaluate matching on two different coordinate planes onto which grains are projected, as shown schematically in Figs. 2 and 3. The coordinates of the centre of mass, and several parameters associated with the morphology, intensity and contrast variations of each diffraction spot are calculated. Information on grain morphology is also imported, in the form of vector coordinates of the positions of grain boundary particles, after applying the GBT process.

There are a number of experimental factors that cause inaccuracies in the DAGT technique, such as blurring of XRD spots, misalignment in and between the XMT and XRD experimental setups, and more importantly, inaccuracy in grain geometry characterisation due to the limited number of grain boundary particles available in practical materials. Therefore, the DAGT technique employs algorithms to calculate two probabilities, $P_{1}$ and $P_{2}$, that quantitatively indicate the likelihood that a given grain has generated a particular diffraction spot. The definition of the first probability, $P_{1}$, is based on whether a diffraction spot is detected when a pencil beam has intersected a grain. Each grain projected onto the $y-z$ plane is outlined for the calculation of $P_{1}$, as shown in Fig. 2, for all the rotation angles, $\omega$. The total number of times that a specific diffraction spot is observed with respect to a specific grain, $N_{\text {diff, }}$ is divided by the total number of times the beam intersects the grain, $N_{\mathrm{GBT}}$, and this determines $P_{1}$ :

$$
P_{1}=\frac{\left(N_{d i f f}\right)}{\left(N_{G B T}\right)} \text {. }
$$

This reflects the spatial consistency between individual diffraction spots and grain morphology on the $y$-z plane. Comparison, in the example of Fig. 2, of the respective locations of the grain and the specific diffraction spots (highlighted by the dashed circles) indicates a $P_{1}$ value of about 0.47 in this instance.

The centre of mass of each diffraction spot is also used, to calculate the point where the diffracted X-ray beam originates on the $x-y$ coordinate plane, derived trigonometrically from the value of $2 \theta$, as shown in Fig. $3 . P_{2}$ is calculated using a negative exponential value of the distance, $d$, between the origin of a given diffraction spot and the centre of mass of the grain of interest, as follows:

$$
P_{2}=e^{-k d}
$$


where $\mathrm{k}$ is a constant. If both the $P_{1}$ and $P_{2}$ values ascribed to the combination of a specific diffraction spot and a particular grain are below predetermined threshold values, connection between the diffraction spot and the grain is rejected.

Although the screening process greatly reduces the number of diffraction spots related to a particular grain, there are still a considerable number of extraneous diffraction spots for each grain, especially when deformed material is analysed. Crystallographic indexing of all the grains is, however, performed at this stage, using the diffraction spots that have passed the first selection process. Crystallographic consistency of angles between diffracting plane normals for diffraction spot pairs is systematically evaluated to eliminate physically inconsistent pairs. Crystallographic orientation is then derived from the scattering vectors of each diffraction spot, $\mathrm{H}_{h k l}$ and $\mathrm{H}_{h^{\prime} k^{\prime} l}$, for each pair of diffraction spots with respect to a given grain, as follows:

$$
\widehat{\mathbf{e}}_{3}=\widehat{\mathbf{e}}_{1} \times \widehat{\mathbf{e}}_{2} \text {, where } \widehat{\mathbf{e}}_{1}=\frac{\mathbf{H}_{h k l}}{\left|\mathbf{H}_{h k l}\right|} \text { and } \widehat{\mathbf{e}}_{2}=\frac{\mathbf{H}_{h k l} \times \mathbf{H}_{h^{\prime} k^{\prime} l^{\prime}}}{\left|\mathbf{H}_{h k l} \times \mathbf{H}_{h^{\prime} k^{\prime} l^{\prime}}\right|} \text {, }
$$

where $\hat{e}_{1}, \hat{e}_{2}$ and $\hat{e}_{3}$ are the unit vectors. The scattering vector is determined from the previously established Millar indices and the angle of rotation, $\omega$, around the $z$-axis defined in Fig. 1.

It is reasonable to assume that a number of diffraction spot pairs are diffracted from each grain, thereby realising the state of oversampling for the determination of crystallographic orientation. Such oversampling can, however, be of benefit in the further sorting of extraneous diffraction spots. An example of such spot sorting is shown in Fig. 4, where all the calculated orientations for a specific grain are plotted on a pole figure. This data has been taken from the specimen that will be discussed below. A total of 32 diffraction spot pairs are agglomerated within narrow regions (i.e., those indicated by the six circles) of $7^{\circ}$ in misorientation angle. The remaining diffraction spot pairs are almost randomly distributed, being unrelated to the grain of interest. In this case, it is reasonable to assume that at least one of any given pairs of diffraction spots is emitted from a different grain. The pencil-beam XRD technique also finds important application in the characterisation of localised deformation (i.e., orientation mapping inside grains), which cannot be obtained using the GBT technique. Deformation inhomogeneity, such as grain distortion, is thereby visualised during plastic deformation. This is exploited later, in Fig. 12. It has been reported that gallium does not penetrate 
[22], or at least penetrates slowly [23], into low-angle grain boundaries. Although it is not demonstrated in the present study, weakness in the detectability of grain boundaries in the GBT technique may potentially be compensated for by employing the pencil-beam XRD technique.

\subsubsection{Processing of blurred diffraction spots due to plastic deformation}

Figure 5 shows an example of blurred diffraction spots, obtained at an applied strain of $27.0 \%$, in the aluminium alloy used in the present study. Both circumferential and radial broadenings are observed in the deformed material, in contrast to the distinct spotty reflections obtained before loading. Furthermore, the significant peak broadening in the circumferential direction clearly demonstrates the sensitivity of the peak profiles to structural changes upon plastic deformation. The profiles are asymmetric in the circumferential direction and highly modulated. The most pronounced example of asymmetry occurred in the case of the (200) peak shown in Fig. 5 (b). The elongated peaks observed after deformation were observed over a rotation angle, $\omega$, of a few degrees, as shown in Fig. 6. In the modified DAGT technique, these consecutively observed spots are labelled together, by calculating the gravity centre of each diffraction spot, as shown in Fig. 6. Such '3D' labelling of diffraction spots enables determination of the average orientation for each grain.

\section{Experimental and image processing details}

\subsection{Synchrotron radiation experiment}

\subsubsection{Materials}

An Al- $\mathrm{Cu}$ binary alloy was melted in an atmosphere of air, and cast into iron moulds. Aluminium of $99.99 \%$ purity and an $\mathrm{Al}-30 \% \mathrm{Cu}$ mother ingot were used to prepare the $\mathrm{Al}-\mathrm{Cu}$ alloy. Cast ingots were homogenised at $823 \mathrm{~K}$ for $18 \mathrm{ks}$, cold rolled by $90 \%$, and given recrystallisation treatment at $803 \mathrm{~K}$ for $45 \mathrm{sec}$; after which, a solution treatment was applied at $768 \mathrm{~K}$ for $18 \mathrm{ks}$, and then a natural aging treatment. The $\mathrm{Cu}$ concentration varied between 3 and 4 mass\% from lot to lot. The samples' stress-strain response, shown in Fig. 7, was roughly the same, though their composition varied. An Al-3mass\% alloy was used in the first trial, for mapping strain (Figs. 13-16). An Al-4mass\% alloy was then used, in the second beam time, one year later, for assessment of the effects of greater applied strain (Figs. 9-12). The alloy exhibited equiaxed grains of 30-200 $\mu \mathrm{m}$. The diameter of the dispersion particles, which play a key role in the DAGT technique, ranged between 1 and $10 \mu \mathrm{m}$. After the heat treatments, 
the samples were cut, by electronic discharge machining, into I-bar shapes, in order to fit into a tensile rig. The specimens had a cross-sectional area of $\sim 600 \times 600 \mu \mathrm{m}$ in the gauge section.

\subsubsection{DAGT experiment}

A DAGT experiment was performed using the X-ray imaging beamline, BL20XU, of SPring-8. A material test rig specially designed for in-situ mechanical tests at synchrotron radiation facilities was positioned approximately $80 \mathrm{~m}$ from the X-ray source. Monochromatic X-ray beams with photon energies of 20 and $25.6 \mathrm{keV}$, generated by a liquid nitrogen-cooled Si (111) double-crystal monochromator, were used for the XMT and XRD experiments, respectively. Two CMOS detectors were placed side by side, with their axes parallel, and alternately utilised to quickly switch between the XMT and XRD experiments. The image detector used in the XMT imaging consisted of a $1440(\mathrm{H}) \times 1920(\mathrm{~V})$ element digital CMOS camera (ORCA-Flash 2.8, Hamamatsu Photonics K.K.), single crystal scintillator $\left(\mathrm{Lu}_{2} \mathrm{SiO}_{5}: \mathrm{Ce}\right)$, and lens. The image detector used for the pencil-beam XRD experiment was a combination of a 2048 $(\mathrm{H}) \times 2048(\mathrm{~V})$ element digital CMOS camera (ORCA-Flash 4.0, Hamamatsu Photonics K.K.), powder scintillator $\left(\mathrm{Gd}_{2} \mathrm{O}_{2} \mathrm{~S}: \mathrm{Tb}\right)$, and lens. The effective pixel size of these detectors was 0.5 and $6.5 \mu \mathrm{m}$, and they were positioned 20 and $14 \mathrm{~mm}$ behind the sample, respectively. The XRD detector registered spots diffracted from the $\{111\}$, $\{200\},\{220\},\{311\},\{222\},\{400\}$ and $\{331\}$ aluminium planes.

A total of 1,800 radiographs, scanning $180^{\circ}$ in $0.1^{\circ}$ increments, were obtained in each XMT scan. Image slices were reconstructed from a series of projections based on the conventional filtered backprojection algorithm [24].

A Fresnel zone plate of $300 \mu \mathrm{m}$ diameter was used for focussing monochromatic $\mathrm{X}$-rays in the XRD experiment. The focal distance and diffraction efficiency of the Fresnel zone plate used are expected to be $960 \mathrm{~mm}$ and $\sim 0.15$ at $20 \mathrm{keV}$, respectively. A first-order diffracted beam was used for creating a pencil beam of $10 \mu \mathrm{m}$ in diameter, by setting an appropriate defocus offset quantity; and 0th-order diffraction was cut off using a centre beam stop. Specimens were rotated through $180^{\circ}$ at each point, with a rotation step of $1^{\circ}$ and an exposure time of $100 \mathrm{msec}$ per degree. After each complete rotation, the pencil beam was moved by $10 \mu \mathrm{m}$, parallel to the $y$ axis, and the specimens were again rotated. This scanning and rotation procedure was repeated until the entire region of interest in the samples $(400(\mathrm{H}) \times 700(\mathrm{~V}) \mu \mathrm{m})$ had been scanned, scanning down the $z$ axis at intervals of $10 \mu \mathrm{m}$.

The first XMT scan was performed without loading, together with the first XRD scan; and the subsequent 6 XMT scans were performed during loading, at a loading rate 
of $0.2 \mathrm{~mm} / \mathrm{sec}$. The strain levels at which the XMT scans were performed are shown in Fig. 7. To suppress image blurring caused by relaxation behaviour during scanning, displacement was fixed at the 6 strain levels for $10 \mathrm{~min}$., before and during each scan. After the 6th scan, which was immediately prior to the final rupture, the second XRD scan was performed, before the liquid gallium wetting technique was applied to the specimen. After applying gallium to the sample surface, specimens were held for 500 sec at $323 \mathrm{~K}$, and then further XMT scanning was performed, to characterise the grain structure in $3 \mathrm{D}$.

\subsection{Image processing}

The Al-Cu alloy contained only equilibrium $\theta-\mathrm{Al}_{2} \mathrm{Cu}$ phase particles as dispersion particles, which are larger than the spatial resolution of the projection-type XMT technique (i.e., $1 \mu \mathrm{m}$ ). The linear absorption coefficient values of the $\theta-\mathrm{Al}_{2} \mathrm{Cu}$, aluminium matrix, and pores, are about $35,11.5$, and below $0 \mathrm{~cm}^{-1}$, respectively, at an X-ray energy of $20 \mathrm{keV}$. The grey value in the XMT images captured before gallium application was calibrated such that the linear absorption coefficients of $0-40 \mathrm{~cm}^{-1}$ fell within an 8-bit grey-scale range between 0 and 255 . The conditions for segmenting the gallium-enhanced grain boundaries were the same as in the previous study [14]. To estimate the volume of each dispersion particle with sub-voxel accuracy, facetted iso-intensity surfaces of pentagonal shape were computed from the volumetric data set, using the Marching Cubes algorithm [25]. To suppress inaccuracies originating from image noise, only particles over 9 voxels in volume were counted as particles in the XMT images. The coefficients $\alpha, \beta$ and $\gamma$, employed in the MTT technique [20], were $0.8,0.1$ and 0.1 , respectively.

In the diffraction patterns obtained from the pencil-beam XRD experiment, only diffraction spots originating from aluminium grains were selected, by adopting only those diffraction spots located within $\pm 50 \mu \mathrm{m}$ of the Debye-Scherrer rings, calculated using the known lattice parameter value of $0.40496 \mathrm{~nm}$. Raw images were processed using background subtraction and thresholding processes. The threshold value varied, experiment by experiment, and thus some trial and error was involved. Only spots over 26 voxels in volume were counted as diffraction spots in the 3D labelling of diffraction spots (described in Section 2.2.3). Diffraction spots with observed $\omega$ of greater than $15^{\circ}$ were eliminated as ring artefacts [26], which are unavoidable in such digital imaging. The $P_{1}$ and $P_{2}$ values (described in Section 2.2.2) were 0.6 and 0.6 , respectively, before loading, but were reduced to 0.2 and 0.6 after loading, in order to adopt grains with severe distortion and/or low-angle boundary formation due to plastic deformation. 
Diffraction spot pairs agglomerated within a $2^{\circ}$ calculated misorientation angle were assumed to indicate the true orientation of a corresponding grain, according to the procedure described in Section 2.2.2. The percentage of grains successfully indexed under the abovementioned conditions decreased from $86 \%$ to $71 \%$ with $27.0 \%$ macroscopic deformation. The unsuccessful measurement was mainly due to small grain size in the initial unloaded state, while the number of diffraction spots had decreased after deformation.

In the present study, the grain-specific Taylor factor was calculated. This factor reflects the plastic response of a grain, which is attributable to the local activity of slip systems and local deviations from a given macroscopic stress state. Here, the factor was calculated in order to compare the propensity for slip deformation among numerous grains, using local strain values and the crystallographic orientation of individual grains. The deformation rate tensor, which is comprised of both normal and shear strain components, was derived from experimental data obtained by employing the MTT technique [20]. The strain values were then averaged for each grain, which was also ascribed its average orientation. The local Taylor factor was then calculated such that five slip systems which had minimised the internal work became operative for an associated strain increment.

\section{Deformation texture formation}

\subsection{Visualised grain structure}

The 3D reconstructed grains are shown before deformation, in Fig. 8, in the form of a grain map. All 23 grains completely embedded in the rectangular parallelepiped of 193 x 93 x $180 \mu \mathrm{m}$ are shown. The 3D visualisation of the grains represents differences in crystallographic orientation. The grain-colour distributions, arising mainly from the recrystallisation and solution treatments applied, display distinct differences among neighbouring grains. Although the morphology of the grains is not equiaxed, it is reasonable to assume that remarkable recrystallisation occurred during the heat treatments. Even small grains, such as Grain $19(11.3 \mu \mathrm{m}$ in length in the longitudinal direction), are visualised with successfully determined crystallographic orientations.

\subsection{Deformation texture formation}

Figure 9, in which the colour scale of individual grains and plots is dependent on crystallographic orientation, shows individual grains over the entire region of interest, before and after plastic deformation of $27.0 \%$. Some grains are intentionally not 
displayed, in order to facilitate the observation of internal grains. To examine the texture evolution during tensile deformation, the average orientations of the respective grains are represented in inverse pole figures, for the longitudinal directions (RD), in Figs. 9 (c) and (d). In the unloaded state, the grains exhibit almost random distribution, and the rolling texture commonly seen in practical sheet aluminium alloys is not observed here. Figs. 9 (b) and (d), which show the distribution of crystallographic orientation after uniaxial tensile deformation, reveal that certain crystallographic axes are almost parallel to the tensile direction. The stereographic triangle of Fig. 9 (d) clearly shows that the orientation space is subdivided into three regions, including grains with their tensile axes near the crystallographic <111> direction, those along the hypotenuse, and those outside these regions. The first and second groups correspond to the deformation texture characteristic to tensile deformation, which has a preferential orientation of $\langle 111\rangle$ and $<112>$ in the case of aluminium alloys [27]. The majority of the grains belonging to the third group are observed in the vicinity of the specimen surface, implying strong free surface effects.

Figure 10 shows the rotation paths, between an applied strain of 0 and $27.0 \%$, for the 22 grains observable on the virtual cross-section shown in Fig. 11. In the case of moderate deformation, it has been reported that rotation paths, as represented in inverse pole figures, are approximately straight [28]. It should be noted, however, that the straight lines in Fig. 10 merely connect the initial and final orientations, due to the lack of intermediate XRD measurements in the present experiment. Seven grains belong to the third group abovementioned, with five of the seven grains located in the vicinity of the specimen surface; the remaining two grains (Grains E and G in Fig. 11) are indicated with dashed ellipses in Fig. 10. It has been reported that orientation rotation behaviour in polycrystalline materials exhibits a clear dependence on initial grain orientation [11]. However, here, the rotation behaviours of the seven grains are not attributable to their initial grain orientation. This is because the majority of the seven grains showed orientation rotation toward directions opposite to the preferential orientations, and not certain stable orientations, such as the $\langle 001\rangle$ orientation reported in the literature $[29,30]$, did not persist during deformation. Such retrograde rotation has also been reported in the literature [30, 31].

It seems fairly reasonable that the grains would respond differently when significant morphological changes are imposed due to large applied strain. It is, however, of great interest to understand how such retrogressive rotation occurs during global texture formation. In Fig. 11, the average strain for each grain is magnified and expressed as a rhombohedral box, to better illustrate the deformation behaviour of the individual grains. 
Each box indicates a grain, and the shape of the box represents the magnitude of all three normal strains and three shear strains. Note that all the strain components have been magnified five times to enhance viewability; the box size is therefore not representative of the absolute size of the respective grain, but simply provides a visual sense of the grain's 3D deformation pattern. It is interesting to note that Grain G, which has rotated even farther away from the common orientations of deformation texture, shows a different deformation pattern than the surrounding grains. In Fig. 11 (b), Grain $\mathrm{G}$ was virtually deformed on the presumption that this grain had been oriented to the nearest [111] direction. This result shows that deformation mismatching is enhanced more than the actual response of Grain G, shown in (a); and this implies that the characteristic rotation behaviour of Grain $\mathrm{G}$ is attributable to consistency in deformation behaviour with surrounding grains. Becker et al. have also reported the effects of grain interaction on orientation evolution, based on their polycrystal simulations [32]. There appear to exist two opposing forces influencing grain rotation: one acting to stabilise orientation with respect to the deformation texture, and another arising from interaction with adjacent grains, which acts to maintain local compatibility and equilibrium. It is natural, however, to assume that the former becomes predominant and the latter gradually diminishes over time [32]. It may therefore be inferred that the characteristic rotation behaviour seen in Fig. 10 may be a transient phenomenon.

\subsection{Local rotation behaviour}

As is well known, most metals and alloys exhibit high-angle grain boundaries after exposure to high temperatures. Low-angle grain boundaries are generated when grains are heavily deformed under external strain. In the present study, as observed in Fig. 4, the calculated orientations were more or less scattered after plastic strain of $27.0 \%$ had been applied. The DAGT technique, therefore, has the potential to resolve in-grain orientation gradients, which may provide detailed information about many aspects of texture and subsequent grain subdivision.

Fig. 12, which illustrates such spatially distributed orientation in a specific grain, was plotted from stacked 2D images of orientation distribution, drawn on the respective $x-y$ cross-sections. The grain shown in Fig. 12 corresponds to Grain A in Fig. 11. Grains

$B$ to $H$ are adjacent to Grain $A$ in this virtual cross-section. Among Grains B to $H$, Grains B, C, D, F and H share relatively wide grain-boundary areas with Grain A. Grains B, C and F have average orientations close to $\langle 112\rangle$, while grains D and H are on average close to $\langle 111\rangle$. Unfortunately, there was no solution obtained for the boundary region between Grains $A$ and $H$. It is interesting to note that the 
crystallographic orientation in the lower left side of Grain A, which is adjacent to Grain $\mathrm{D}$, is different from the other regions; and at the same time, this region exhibits intense shear strain, as shown in Fig. 12 (b). Another region where intense shear localisation is observed in Fig. 12 (b) is adjacent to Grain B, which has already been examined in Figs. 10 and 11.

It is reasonable to assume that the amplitude of shear strain on an individual slip system varies within the grain, depending on localised rotation and strain state [33]. It is also to be noted that grain subdivision, where the orientation domains had almost constant crystallographic orientations, were not detected, as shown in Figs. 4 and 12. The internal orientation gradient is observable in Fig. 12. Wert et al., employing the EBSD technique to analyse a cross-section, have suggested the existence of domains emanating from grain corners merging near the centre of a grain, in $\mathrm{Cu}$ deformed up to $15 \%$ in tension [33]. 3D observation (as in Fig. 12), in which a more complex, 3D grain shape is observable than in conventional $2 \mathrm{D}$ observation, has revealed the effects of inhomogeneous and strong interactions with selected surrounding grains.

\section{Localised deformation behaviour}

\subsection{Analyses involving grain-averaged quantities}

Although Fig. 12 demonstrates that the strain distribution within individual grains can be highly heterogeneous, grain-averaged quantities were intentionally evaluated in the conventional manner. The mean equivalent strain and the respective ratios between neighbouring grains were plotted as functions of either Taylor or Schmid factors, in Fig. 13, and no clear tendency in the relationships is immediately observable. Closer inspection reveals that the mean equivalent strain may be roughly in inverse proportion to the local Taylor factor in (a), if exceptional data such as that for Grains I and $\mathbf{J}$ were eliminated (Grain I is smaller than the pencil-beam size, and Grain Group J falls within the top $15 \%$ in terms of the local Taylor-factor ratio between neighbouring grains). It is, however, impossible to provide unambiguous evidence for such a correlation, based on the figure alone. Such poor correlation is, of course, attributable to localised deviation from the grain-averaged stress state in individual grains, which may arise through grain interactions.

\subsection{Local 3D deformation behaviours}

Strain mapping of the $y-z$ virtual cross-section indicated in the 3D perspective view of Fig. 14 (a) (i.e., the same grain group as shown in Fig. 8) is demonstrated in Fig. 14 
(b)-(i). Local Taylor-factor and Schmid-factor values are also shown, as variously sized red and blue circles, respectively, in Fig. 14 (b). As already observed in Fig. 12 (b), the strain map reveals highly heterogeneous strain in all the strain components, making clear that the grain-averaged evaluation cannot be representative of the deformation mechanism. The majority of the high-strain regions appear to emanate from grain boundaries, which are indicated with black lines. Only high-strain regions have been extracted in Fig. 15 (See also the corresponding animation of Fig. 15). High-strain regions a-h are located along grain boundaries, whilst high-strain regions $i$ and $j$ are located inside Grains 9 and 21, respectively. The reason why no high-strain region is observed between Grains 9 and 21 is that the grain boundary between these grains has a misorientation angle of only $0.8^{\circ}$. Another low-angle boundary lies between Grains 9 and 11 , which has a misorientation angle of $14.7^{\circ}$. Such low-angle boundaries exhibit continuous strain distribution between grains, without exception, in Fig. 14.

It also appears, from Fig. 14, that the high-strain regions tend to be located between grains with large differences in Taylor factor, where additional slip systems may be activated for coherency with neighbouring grains. The heterogeneity of the deformation is attributable to strong interactions among anisotropic grains of different size and shape, which cause significant distortion of individual grains, as has been shown by Xhao et al., through their 3D crystal plasticity finite element simulation [34]. However, it does not appear that through-sample strain percolation follows kinematically soft grains while avoiding kinematically hard grains, as has been reported by Raabe et al., through their plane-strain crystal plasticity finite element simulation [35]. It has also been suggested that material near grain boundaries mediates incompatibility with adjacent grains, whereas material away from such boundaries can deform in a more arbitrary manner, thereby resulting in grain subdivision [33]. However, the existence of such mantle-like structures is not observable in Fig. 14.

It is also interesting to note that the 10 extracted grains in Fig. 14 appear to form two groups in terms of lateral strains, $\varepsilon_{x x}$ and $\varepsilon_{y y}$, and shear strains, $\gamma_{x y}$ and $\gamma_{y z}$. For example, Grains 8, 13, 18 and part of Grain 7 exhibit Poisson deformation only in the $x$ direction, while compressive strain is small in the $y$ direction; whereas the other six grains exhibit the opposite tendency, a combination of small $\varepsilon_{x x}$ and large $\varepsilon_{y y}$. Note that the grains in each grain group are separated by high-angle grain boundaries. Such coordinated deformation among multiple grains is more obvious in the shear strain components. For example, the $\gamma_{y z}$ component shows that Grains 7, 8 and 18 are sheared in the negative $z$ direction, while Grains $1,3,9,11$ and 21 are displaced in the positive $z$ direction. It is also notable that the grains constituting the grain groups seem slightly 
different, component by component. For example, Grain 4 is sheared with Grains 7 and 8 in $\gamma_{y z}$, whereas it is coordinated with surrounding Grains 3 and 9 in $\gamma_{x y}$.

Although not mentioned in the previous section, such behaviour can also be observed in the different representation of grains for the different specimen, as shown in Fig. 11 (a). There, for example, Grains A, C, D, H, J, K, O and P have fairly large negative $\gamma_{y z}$ values, whereas Grains $\mathrm{G}$ and I are sheared in the opposite direction. Grains $\mathrm{B}, \mathrm{L}, \mathrm{M}$ and $\mathrm{N}$ have negligible shear strains in all three directions. In addition, neighbouring grain pairs sometimes show analogous deformation behaviours, as in the case of Grains B and N, and Grains C and D.

In the conventional Taylor-type models for polycrystalline plasticity, it is assumed that individual grains deform as units, undergoing, as a consequence, the same macroscopic shape change [36]. In some modified Taylor-type models, such as the LAMEL model, common shear relaxations are assumed between two neighbouring grains, in order to obtain better agreement between prediction and experiment by relaxing the strong compatibility hypothesis of the original Taylor model. Grain boundary sliding is prevented by introducing such an assumption, resulting in equal and opposite shear deformations (i.e., net near-zero shear across the grain boundary plane) in two neighbouring grains [37]. This implies that, as has been demonstrated by Kanjarka et al., through their 2D crystal plasticity simulation, two adjacent grains undergo macroscopically applied deformation better than individual grains [38]. The characteristic grouping of deforming grains observed in the present study implies that such inevitable relaxation occurs on a more mesoscopic scale than is expected in real materials. Recently, more advanced texture models, such as the GIA model [39] and the RGC model [40], which take interaction among multi-grains into account by assuming the generation of geometrically necessary dislocations, have been developed and applied to similar issues. Such predictions have improved the prediction of anisotropic deformation behaviour. Comparison with such models or 3D image-based crystal plasticity simulations [34] is inevitable to understand the characteristic deformation behaviour.

The evolution behaviour of local shear strain is shown in Fig. 16. Small regions of elevated local shear strain seem observable in Grains 7,8 and 9, at the macroscopic applied strain of $2.8 \%$ (Fig. 16 (a)). The blue patches in Grains 7 and 8 merge across the common grain boundary (Fig. 16 (b)). A region along the triple-junction line among Grains 9, 18 and 21 has also become severely strained (Fig. 16 (b)), after strain localisation was observed between Grains 4 and 9 in (a). As a consequence, the two adjacent grain groups with different shear directions, shown in Fig. 14 (f), have been 
formed. It has been argued that deformation bands extend into the centre of grains from the grain boundary, due to the influence of neighbouring grains [33]. It is clear that actual grain behaviours is often not straightforward due to the effects of complex interaction among grains.

Quadruple junction points, as in Fig. 17 (a), were sometimes observed. It is worth noting that hydrostatic strain is relatively intensive around such quadruple junction points, as shown in Fig. 17 (b). In Fig. 17 (c), the local strain values in parts of four grains (defined by the white dashed square in Fig. 17 (b)) are sampled and expressed as rhombohedral boxes (as in Fig. 11), to better illustrate the deformation behaviours in the vicinity of the quadruple junction point. As in Fig. 11, all the strain components have been magnified five times to enhance viewability. The figure shows that all four grains have some positive $\gamma_{y z}$, with Grain 9 far exceeding the other three grains in this respect. This strong mismatching has generated a large gap at the quadruple junction point in the box model, which implies that a large hydrostatic component has been generated in the actual material. This result qualitatively coincides with the modelling approach of Zisman et al., in which they demonstrated, through their 2D semi-continuous model, an inhomogeneous shear stress component and resultant strong singularity of hydrostatic stress state around a triple junction line [41]. Interestingly, here the large hydrostatic strain covers a wide area, extending deeply into Grain 13, which has a relatively small volume compared to the average grain size. Since damage evolution is strongly associated with stress triaxiality, this implies that damage enhancement can be extensively triggered by such localised grain-grain interaction.

\section{Summary and concluding remarks}

The diffraction-amalgamated grain boundary tracking (DAGT) technique has been successfully developed by combining the grain boundary tracking technique with a pencil-beam X-ray diffraction technique. DAGT has been applied to the tensile deformation of $\mathrm{Al}-\mathrm{Cu}$ alloys, yielding a variety of results with implications to ductile deformation and texture formation in polycrystalline materials. Among other things, the DAGT technique enables experimental investigation of the various phenomena attributable to the complex and heterogeneous structures of actual polycrystals.

Texture evolution during tensile deformation of $27 \%$ in plastic strain has been successfully characterised with the DAGT technique. The characteristic rotation behaviours of a limited number of grains were observed to exhibit orientation rotation toward directions opposite to preferential orientations such as $\langle 111\rangle$. Such exceptional 
rotation behaviours were interpreted in terms of the 3D deformation behaviour of individual grains. It is suggested that one force influencing grain rotation, which arises from interaction with adjacent grains, competes with another, acting to stabilise orientation with respect to deformation texture. Both the orientation and plastic strain distribution of individual grains have provided quantitative evidence for the effects of strong grain-grain interactions, including those in the vicinity of quadruple junction points. 3D strain mapping has revealed highly heterogeneous strain distribution in grains, which undermines conventional grain-averaged evaluation of polycrystalline plasticity. Interpretation of the heterogeneous strain distribution was not straightforward, however; neither strain percolation through kinematically soft grains, nor mantle-like structure in individual grains, was observed. Instead, characteristic coordinated deformation among multiple grains was observed, especially in terms of the shear strain components, which suggested the existence of such relaxation behaviours, on a mesoscopic scale, in actual materials.

A complete discussion of local deformation mechanisms would be beyond the scope of this paper. A large-scale (both in grain number and degrees of freedom inside grains) crystal plasticity simulation, in which the actual topology of grains in practical materials is simulated in $3 \mathrm{D}$, will be a matter for future study. We can expect to evaluate the contribution of grain-grain interaction in comparison to the more intrinsic effects of initial orientations [40], including how in-grain subdivision during plastic deformation coincides with observed mesoscopic deformation patterns.

\section{Acknowledgements}

The SR experiment was performed with the approval of JASRI, through proposal numbers 2013B1027 and 2013A1537. This work was undertaken partly with the support of a Grant-in-Aid for Scientific Research (S) from JSPS, through subject No. 24226015. HT also appreciates the financial assistance of the Light Metal Educational Foundation.

\section{Reference}

[1] V.L. Hein and F. Erdogan, Stress singularities in a two material wedge, Int. J. Fract. Mech., 7 (1971) 317-330.

[2] J.-Y. Buffiere, E. Maire, P. Cloetens, G. Lormand and R. Fougeres, Characterization of internal damage in a MMCp using X-ray synchrotron phase contrast microtomography, Acta Mater. 47 (1999) 1613-1625.

[3] D.J. Dingley, V.Randle, Microtexture determination by electron back-scatter diffraction, J. of Mater. Sci., 27 (1992) 4545-4566. 
[4] H.F. Poulsen, Three Dimensional X-Ray Diffraction Microscopy. Springer, 2004.

[5] U. Lienert, S. F. Li, C. M. Hefferan, J. Lind, R. M. Suter, J. V. Bernier, N. R. Barton, M. C. Brandes, M. J. Mills, M. P. Miller, B. Jakobsen, W. Pantleon, High-energy diffraction microscopy at the advanced photon source, JOM, 63 (2011) 70-77.

[6] W. Ludwig, S. Schmidt, E.M. Lauridsen, H.F. Poulsen, X-ray diffraction contrast tomography: a novel technique for three-dimensional grain mapping of polycrystals. I. Direct beam case, Appl. Crystall., 41 (2008) 302-309.

[7] B.C. Larson, W. Yang, G. Ice, J. Budai, J. Tischer, Three-dimensional X-ray structural microscopy with submicrometre resolution, Nature, 415 (2002) 887-890.

[8] S. Schmidt, S.F. Nielsen, C. Gundlach, L. Margulies, X. Huang, D. Juul Jensen, Watching the Growth of Bulk Grains During Recrystallization of Deformed Metals, Science, 305 (2004) 229-232

[9] S.O. Poulsen, E.M. Lauridsen, A. Lyckegaard, J. Oddershede, C. Gundlach, C. Curfs, D. Juul Jensen, In situ measurements of growth rates and grain-averaged activation energies of individual grains during recrystallization of $50 \%$ cold-rolled aluminium, Scripta Mater. 64 (2011) 1003-1006

[10] L. Margulies, G. Winther and H.F. Poulsen, In situ measurement of grain rotation during deformation of polycrystals, Science, 291 (2001) 2392- 2394.

[11] H.F. Poulsen, L. Margulies, S. Schmidt and G. Winther, Lattice rotations of individual bulk grains: Part I: 3D X-ray characterization, Acta Mater., 51 (2003) 3821-3830.

[12] J. Oddershede, S. Schmidt, H.F. Poulsen, H.O. Sørensen, J. Wright, and W. Reimers, Determining grain resolved stresses in polycrystalline materials using three-dimensional X-ray diffraction, J. Appl. Cryst. 43 (2010) 539-549.

[13] H. F. Poulsen, J. of Appl. An introduction to three-dimensional X-ray diffraction microscopy, Crystallography, 45 (2012) 1084-1097.

[14] H. Toda, Y. Ohkawa, T. Kamiko, T. Naganuma, K. Uesugi, A. Takeuchi, Y. Suzuki, M. Kobayashi, Grain boundary tracking: A four-dimensional visualization technique for determining grain boundary geometry via local strain mapping, Acta Mater. 61 (2013) $5535-5548$.

[15] H. Toda, Z.A.B. Shamsudin, K. Shimizu, K. Uesugi, A. Takeuchi, Y. Suzuki, M. Nakazawa, Y. Aoki, M. Kobayashi, Cavitation during high-temperature deformation in Al-Mg alloys, Acta Mater., 61 (2013) 2403-2413

[16] H. Toda, D. J. LeClere, T. Kamiko, Y. Suzuki, A. Takeuchi, K. Uesugi and M. Kobayashi, Development of diffraction-amalgamated grain-boundary tracking technique and its application to polycrystalline metals, Proc. of SPIE, Developments in 
X-Ray Tomography VIII, edited by S. R. Stock, 8506 (2012) 85060B-1-8.

[17] H. Li, H. Toda, K. Uesugi, A. Takeuchi, Y. Suzuki and M. Kobayashi, Application of Diffraction-Amalgamated Grain Boundary Tracking to Fatigue Crack Propagation Behavior in High Strength Aluminum Alloy, Mater. Trans., 56 (2015) 424-428.

[18] S. Masuda, H. Toda, S. Aoyama, S. Orii, S. Ueda, M. Kobayashi, Effects of sub-surface defects newly found in tempered aluminum alloy die-castings on fatigue properties. J. JFS. 81 (2009) 312-319. (in Japanese)

[19] E. Pereiro-Lopez, W. Ludwig, D. Bellet, J. Baruchel, Grain boundary liquid metal wetting: A synchrotron micro-radiographic investigation, Nucl. Instr. and Methods in Phys. Res. B, 200 (2003) 333-338

[20] M. Kobayashi, H. Toda, Y. Kawai, T. Ohgaki, K. Uesugi, D. S. Wilkinson, T. Kobayashi, Y. Aoki, M. Nakazawa, High-density three-dimensional mapping of internal strain by tracking microstructural features, Acta Mater., 56 (2008) 2167-2181.

[21] C. Hammond, The basics of crystallography and diffraction, IUCr Texts on Crystallography, No. 3, Oxford University Press, Oxford, 1997.

[22] W. Ludwig, E. Pereiro-López, D. Bellet, In situ investigation of liquid Ga penetration in Al bicrystal grain boundaries: grain boundary wetting or liquid metal embrittlement?, Acta Mater., 53 (2005) 151-162.

[23] R.C. Hugo, R.G. Hoagland, The kinetics of gallium penetration into aluminum grain boundaries - in situ TEM observations and atomistic models, Acta Mater., 48 (2000) 1949-1957.

[24] G.T. Herman, Image reconstruction from projections: the fundamentals of computerised tomography. Academic Press, Orland, 1980.

[25] W.E. Lorensen and H.E. Cline, Marching cubes: A high resolution 3D surface construction algorithm, Computer graphics, 21 (1987) 163-169.

[26] F.E. Boas and D Fleischmann, CT artifacts: causes and reduction techniques, Imaging Med., 4 (2012) 229-240.

[27] A.B. Lopes, F. Barlat, J.J. Gracio, J.F.F. Duarte, E.F. Rauch, Effect of texture and microstructure on strain hardening anisotropy for aluminum deformed in uniaxial tension and simple shear, Int. J. of Plasticity, 19 (2003) 1-22.

[28] H.F. Poulsen, L. Margulies, S. Schmidt, G. Winther, Lattice rotations of individual bulk grains: Part I: 3D X-ray characterization, Acta Mater., 51 (2003) 3821-3830.

[29] G. Winther, L. Margulies, S. Schmidt, H.F. Poulsen, Acta Mater., 52 (2004), 863-2872. 
[30] J.-H. Han, K.-K. Jee, K.H. Oh, Orientation rotation behavior during in situ tensile deformation of polycrystalline 1050 aluminum alloy, Inter. J. of Mech. Sci., 45 (2003) 1613-1623.

[31] K.H. Kim, Y.M. Koo, In-situ X-ray diffraction study of single-slip-conditioned copper single crystals during uniaxial deformations, Philos. Mag. A, 81 (2001) 479488.

[32] R. Becher and S. Panchanadeeswaran, Effects of grain interactions on deformation and local texture in polycrystals, Acta Metall. Mater., 43 (1995) 2701-2719.

[33] J.A. Wert, X. Huang, G. Winther, W. Pantleon, and H.F. Poulsen, Revealing deformation microstructures, Materials Today, 10 (2007) 24-32.

[34] Z. Zhao, S. Kuchnicki, R. Radovitzky, A. Cuitiño, Influence of in-grain mesh resolution on the prediction of deformation textures in fcc polycrystals by crystal plasticity FEM, Acta Mater., 55 (2007) 2361-2373.

[35] D. Raabe, M. Sachtleber, Z. Zhao, F. Roters and S. Zaefferer, Micromechanical and macromechanical effects in grain scale polycrystal plasticity experimentation and simulation, Acta Mater. 49 (2001) 3433-3441.

[36] G.I. Taylor, Plastic strains in metals, J. Inst. Metals, 62 (1938) 307-324.

[37] P.V. Houtte, S. Li, M. Seefeldt, L. Delannay, Deformation texture prediction: from the Taylor model to the advanced Lamel model, Int. J. of Plasticity, 21 (2005) 589-624. [38] A.K. Kanjarla, P.V. Houtte, L. Delannay, Assessment of plastic heterogeneity in grain interaction models using crystal plasticity finite element method, Int. J. of Plasticity, 26 (2010) 1220-1233.

[39] O. Engler, M. Crumbach, S. Li, Alloy-dependent rolling texture simulation of aluminium alloys with a grain-interaction model, Acta Mater., 53(2005), 2241-2257.

[40] P. Eisenlohr, D.D. Tjahjanto, T. Hochrainer, F. Roters, D. Raabe, Comparison of texture evolution in fcc metals predicted by various grain cluster homogenization schemes, Inter. J. of Mater. Res., 100 (2009), 500-509.

[41] A.A. Zisman and V.V. Rybin, Mesoscopic stress field arising from the grain interaction in plastically deformed polycrystals, Acta Mater., 46 (1998) 457-464. 


\section{Caption list}

Fig. 1 Schematic diagram of the setup for the pencil-beam XRD experiments.

Fig. 2 Schematic illustration of coupling grains, obtained with the GBT technique; and diffraction spots obtained in the pencil-beam X-ray diffraction experiment. Each grain is projected onto the $y-z$ plane defined in Fig. 1. A probability function, $P_{1}$, is defined, to determine to what extent each consecutively observed diffraction spot overlaps with a specific grain on the detector $(y-z)$ plane.

Fig. 3 Schematic illustration of coupling the position of a specific diffraction spot, back-projected to its original position, and grains (Grains $A-E$ ), obtained with the GBT technique. The origin of the diffraction spot is indicated by the blue ' $x$ '. The extent of the match between the back-projected position and the midpoints of the grain/beam interactions (green ' $x$ ' marks) is determined by defining the second probability function, $P_{2}$.

Fig. 4 An example of the crystallographic orientation calculation for a specific grain; 32 different orientations were calculated. The orientation with the highest number of accumulated spot pairs was adopted as the true orientation for the current grain, while the remaining 31 (non-existent) orientations were calculated by pairing diffraction spots originating from two different grains. A total of 32 spot pairs were accumulated in this case, within a misorientation of 2 degrees.

Fig. 5 Examples of X-ray diffraction spots obtained in the pencil-beam X-ray diffraction experiment: (a) Sharp dots observed before loading are (b) notably blurred after plastic strain of $27.0 \%$ has been applied.

Fig. 6 Schematic illustration of diffraction spots from aluminium, observed consecutively over 5 degrees in $w$ (see Fig. 1). The 3D gravity centre (orange disk) is defined in order to accurately match individual diffraction spots and the respective grains.

Fig. 7 Stress-strain curve obtained in the DAGT experiment.

Fig. 8 Results of the DAGT analysis, representing the appearance and orientations of 23 grains located in a unit box of $193 \mathrm{~mm}$ x $180 \mathrm{~mm}$ x $93 \mathrm{~mm}$. The grain colours represent locations in the inverse pole figure: [001] oriented grains are red, [101] green, and [111] blue (as a legend is available in Fig. 9).

Fig. 9 Results of the DAGT analysis, representing the distributions of crystallographic orientations before and after loading of $27.0 \%$ in plastic strain. Note that strong tension texture is formed after deformation. The colours of grains and inverse pole markings represent locations in the inverse pole figures: [001] oriented grains are red, [101] green, and [111] blue. 
Fig. 10 Changes in orientation up to loading of $27.0 \%$ in plastic strain. The circled arrow shows the rotation of Grain G (highlighted in Fig. 11).

Fig. 11 Representation of mean strain in individual grains, using unit boxes. A regular hexahedron located near the coordinate axis was deformed with the mean strain value of the respective grain, and positioned on a virtual cross-section, in (a). Note that strain values have been magnified five times to enhance visualisation. In (a), the deformed box highlighted in brown corresponds to Grain G, which was rotated, during deformation, even farther away than its initial location from the orientations of deformation texture shown in Figs. 9 and 10. In (b), only this box of interest (now highlighted in green) was virtually deformed, on the presumption that this grain was oriented to the nearest [111] direction.

Fig. 12 Results of the DAGT analysis, representing the 3D distribution of crystallographic orientation in a grain (in (a)), and the corresponding 3D distribution of shear strain, $g_{x y}$ (in (b)). The colours in (a) represent locations in the inverse pole figure. Note that the colour orientation is more enhanced in (a) than in Figs. 8 and 10, in order to express subtle differences in orientation.

Fig. 13 Relationships between various crystallographic parameters and mean or maximum equivalent strain. In (c), the ratios of these parameters between neighbouring grains are plotted.

Fig. 14 Strain maps on a $y-z$ virtual cross-section (shown in (a)). Local plastic strain was calculated between applied strain, $e_{\mathrm{a}}$, of 0 and $10.4 \%$. Plotted are: (b) equivalent strain, $e_{e q}$; (c) normal strain in the $x$ (RD) direction, $e_{x x}$; (d) normal strain in the $y$ (ND) direction, $e_{y y}$; (e) normal strain in the $z$ (TD) direction, $e_{z z}$; (f) shear strain in the $x-y$ direction, $g_{x y} ;(\mathrm{g})$ shear strain in the $y-z$ direction, $g_{y z} ;(\mathrm{h})$ shear strain in the $z-x$ direction, $g_{z x}$; and (i) hydrostatic strain, $e_{m}$. Circle size in (b) denotes the magnitude of the local Taylor factor (red) and Schmid factor (blue). G1-G21 refer to the grains labelled in Fig. 8.

Fig. 15 3D representations of high-strain regions, A-I, in three grains (Grains 6, 9 and 21). Regions with $e_{\mathrm{a}}$ greater than $20 \%$ are highlighted in red. See also the corresponding animation.

Fig. 16 Change in local shear strain $\left(g_{x y}\right)$ distribution, viewed on the same $y-z$ virtual cross-section as in Fig. 14. Local plastic strain was calculated between $e_{\mathrm{a}}$ of 0 and $2.8 \%$ in (a), and 2.8 and $10.8 \%$ in (b). Note the difference in contour levels between (a) and (b).

Fig. 17 Representation of the mean strain in local regions of four neighbouring grains (defined by the dashed white box in (b)), with a unit box around the quadruple junction 
point. Local plastic strain was calculated between applied strain, $e_{\mathrm{a}}$, of 0 and $10.4 \%$. A regular hexahedron was deformed with the mean strain value of the specified region in each grain, as was demonstrated in Fig. 12. Note that the strain values have been magnified five times to enhance visualisation. The four grains in (a) are also shown in Figs. 14 and 15, and the hydrostatic strain distribution in Section A of (a) is shown in (b). 


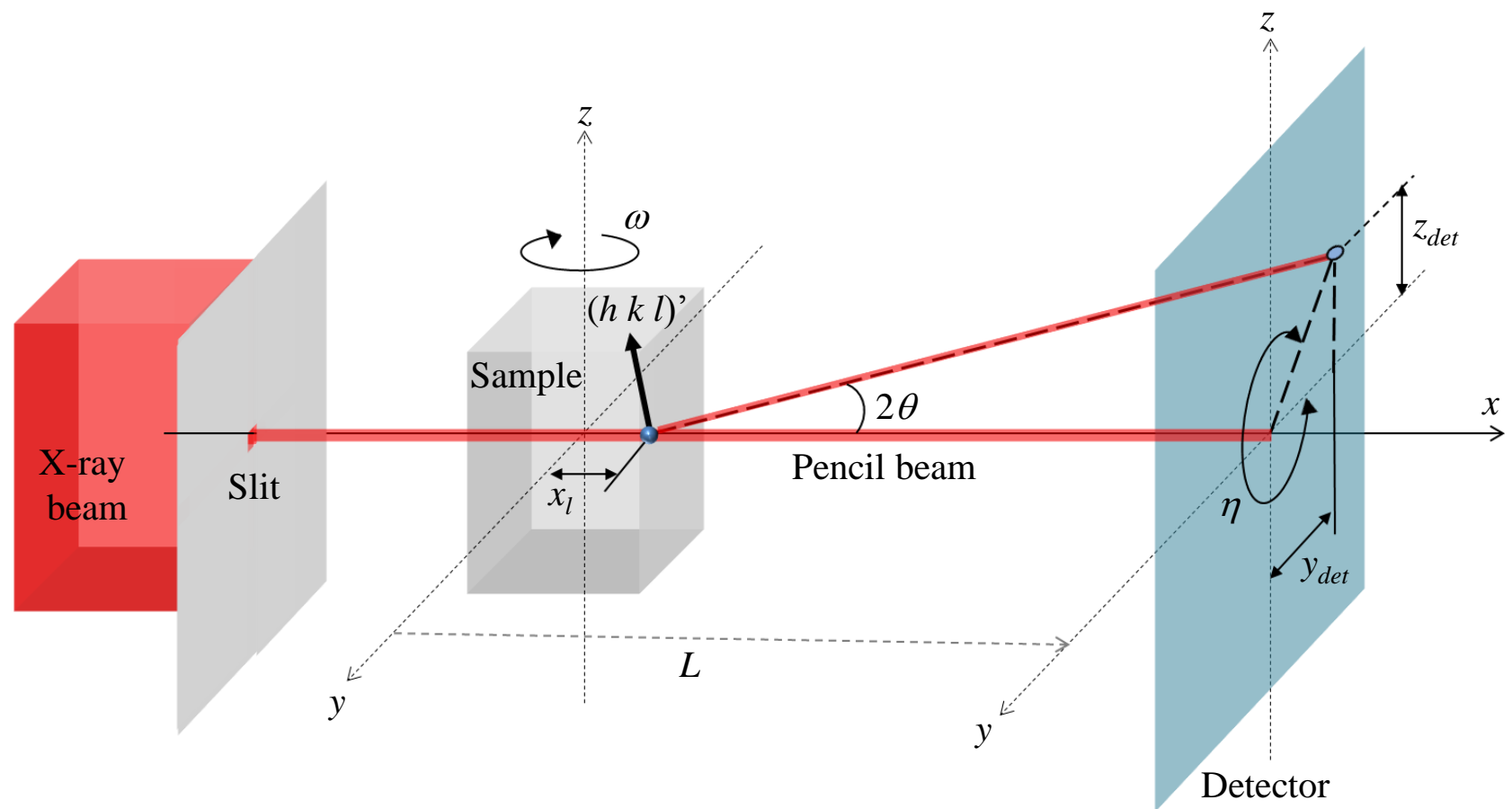

Fig. 1 Schematic diagram of the setup for the pencil-beam XRD experiments. 

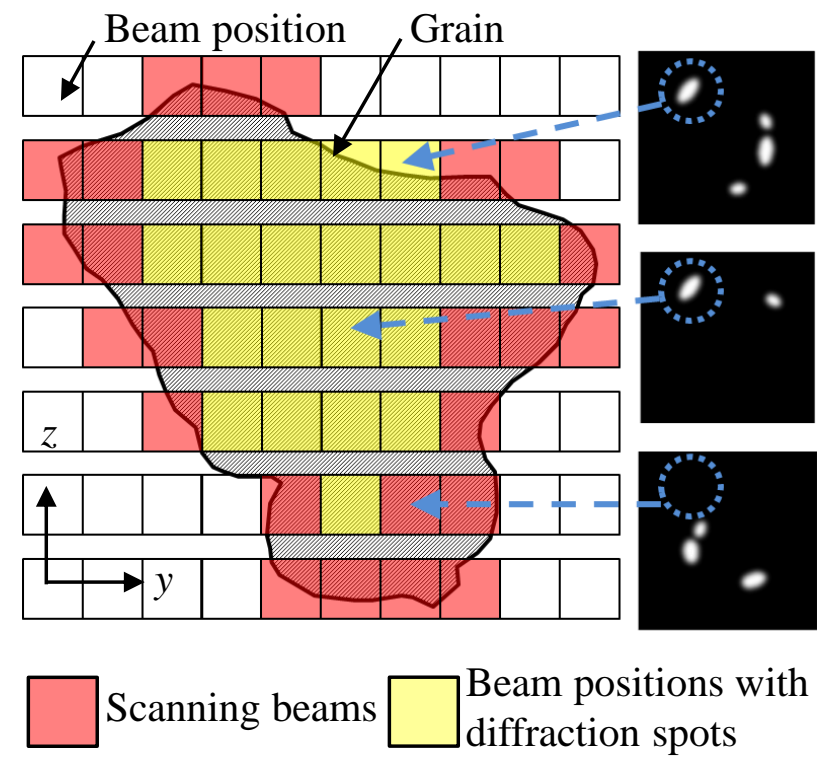

Fig. 2 Schematic illustration of coupling grains, obtained with the GBT technique; and diffraction spots obtained in the pencil-beam $\mathrm{X}$-ray diffraction experiment. Each grain is projected onto the $y-z$ plane defined in Fig. 1 . A probability function, $P_{1}$, is defined, to determine to what extent each consecutively observed diffraction spot overlaps with a specific grain on the detector $(y-z)$ plane. 


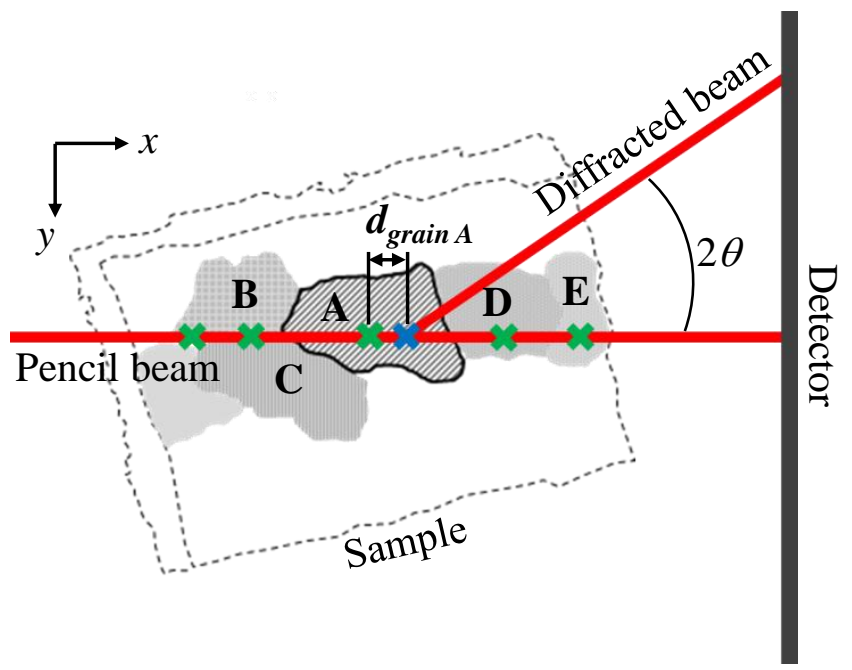

Fig. 3 Schematic illustration of coupling the position of a specific diffraction spot, backprojected to its original position, and grains (Grains A - E), obtained with the GBT technique. The origin of the diffraction spot is indicated by the blue ' $x$ '. The extent of the match between the back-projected position and the midpoints of the grain/beam interactions (green ' $\mathrm{x}$ ' marks) is determined by defining the second probability function, $P_{2}$. 


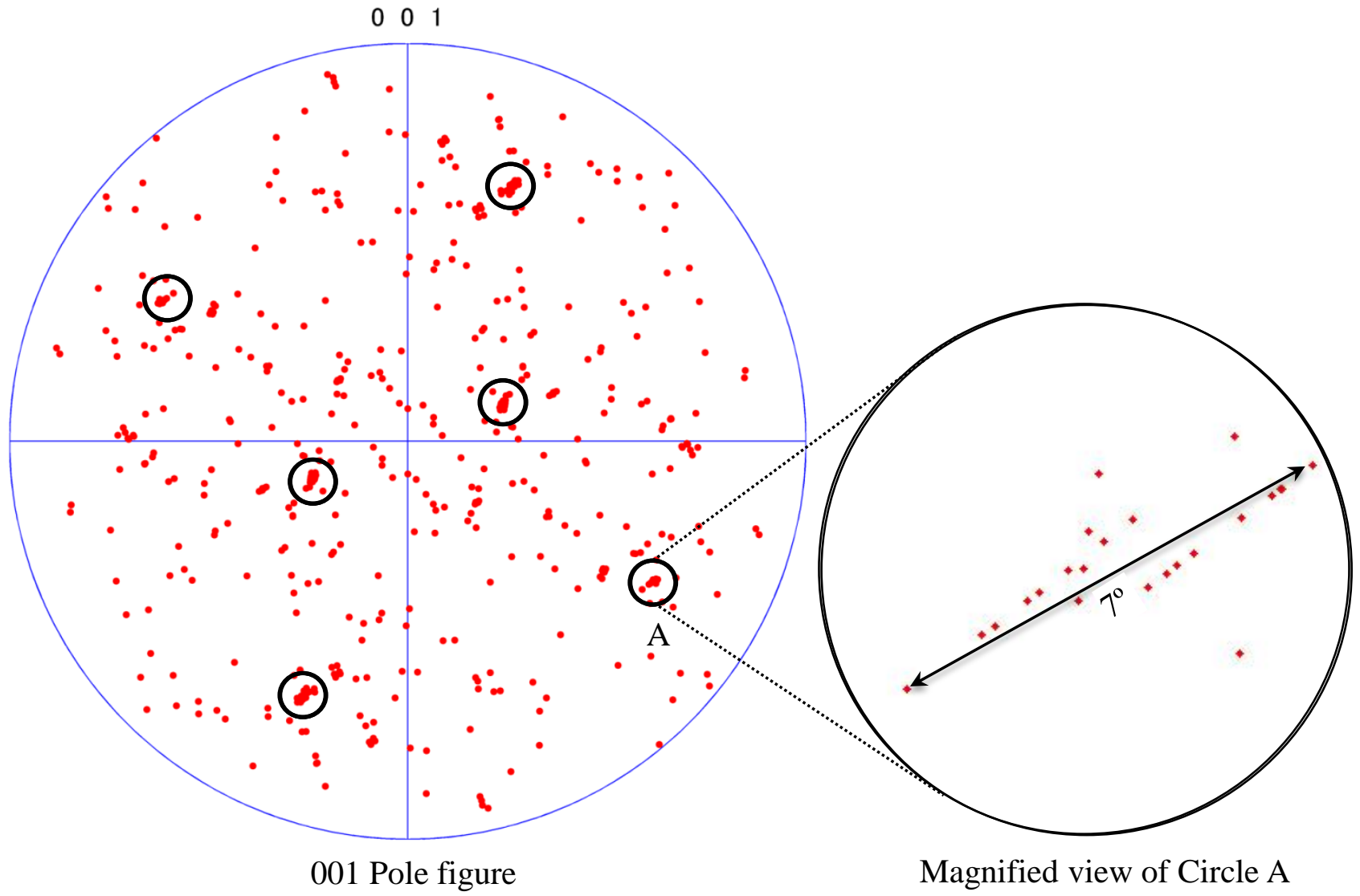

Fig . 4 An example of the crystallographic orientation calculation for a specific grain; 32 different orientations were calculated. The orientation with the highest number of accumulated spot pairs was adopted as the true orientation for the current grain, while the remaining 31 (non-existent) orientations were calculated by pairing diffraction spots originating from two different grains. A total of 32 spot pairs were accumulated in this case, within a misorientation of 2 degrees. 


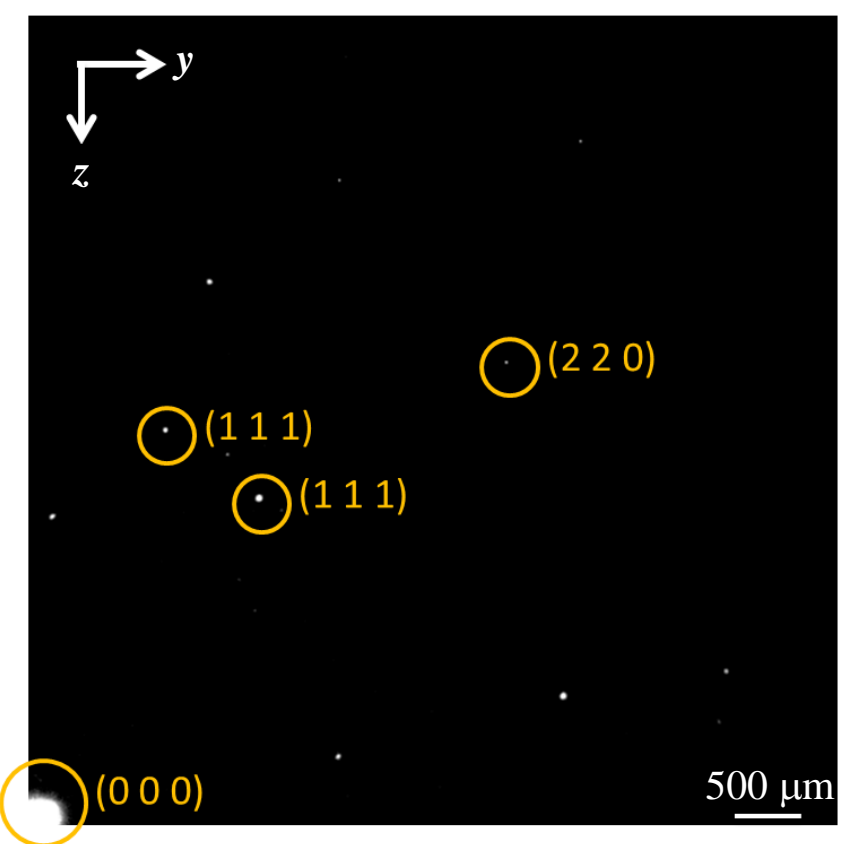

(a) Before deformation



(b) After deformation of $27.0 \%$

Fig. 5 Examples of X-ray diffraction spots obtained in the pencil-beam X-ray diffraction experiment: (a) Sharp dots observed before loading are (b) notably blurred after plastic strain of $27.0 \%$ has been applied. 


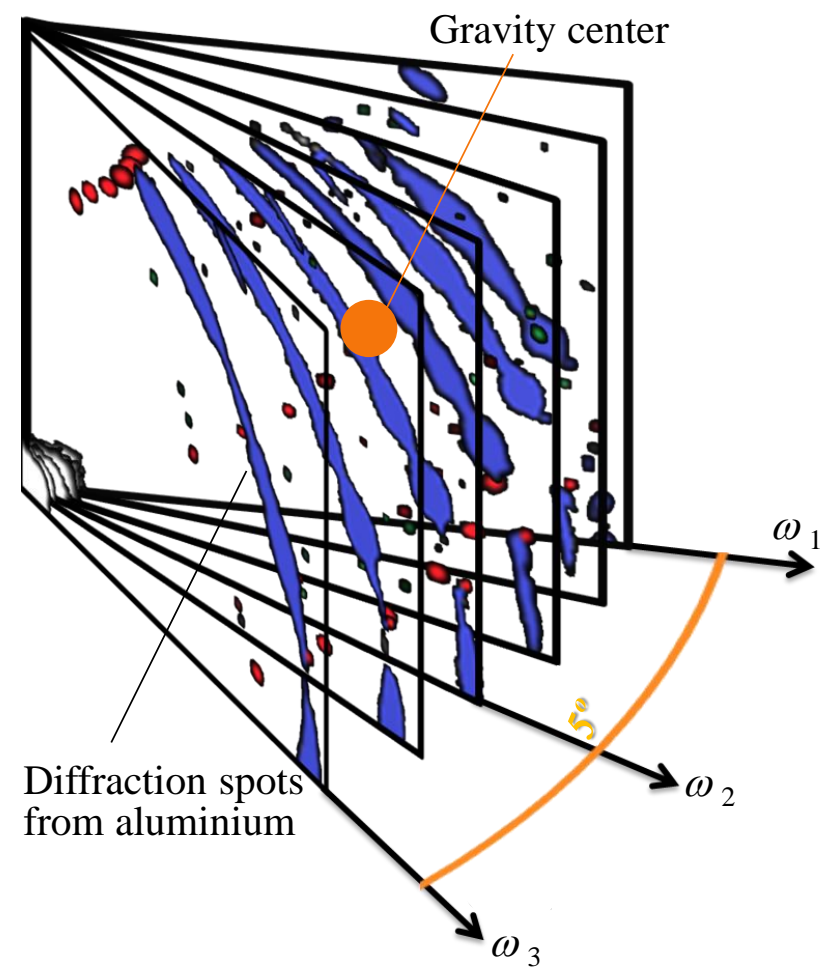

Fig. 6 Schematic illustration of diffraction spots from aluminium, observed consecutively over 5 degrees in $\omega$ (see Fig. 1). The 3D gravity centre (orange disk) is defined in order to accurately match individual diffraction spots and the respective grains. 


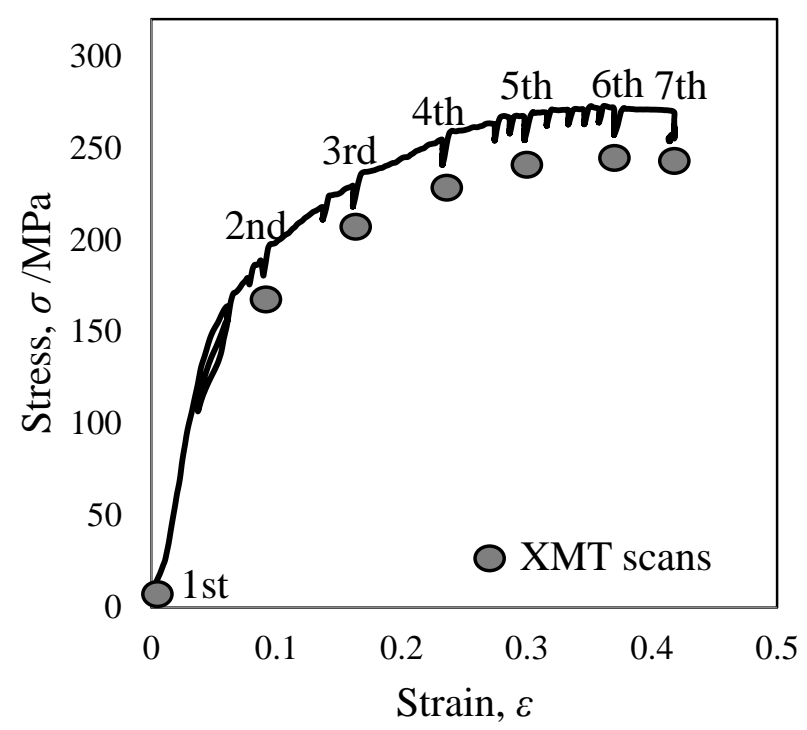

Fig. 7 Stress-strain curve obtained in the DAGT experiment. 


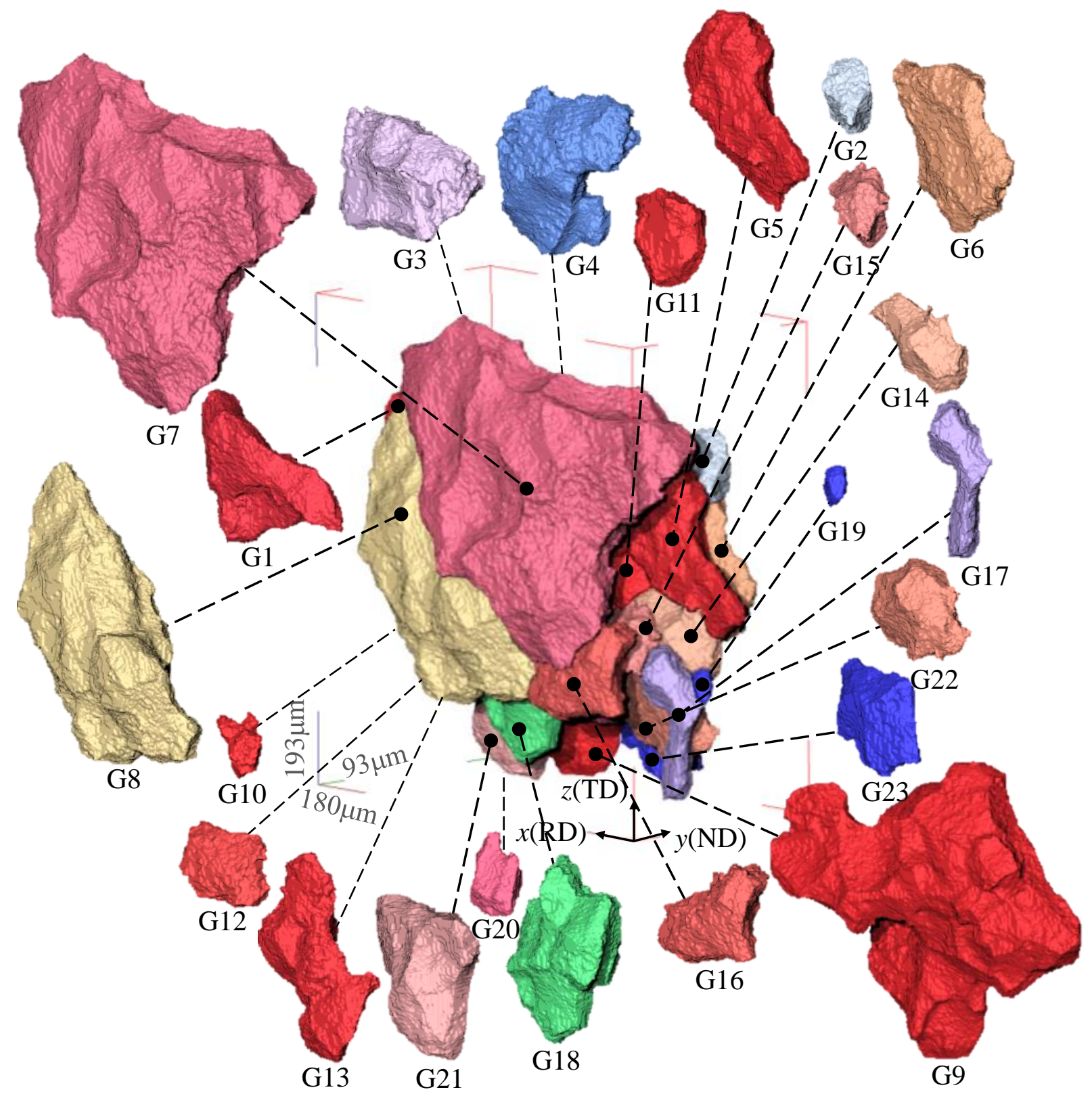

Fig. 8 Results of the DAGT analysis, representing the appearance and orientations of 23 grains located in a unit box of $193 \mu \mathrm{m} \times 180 \mu \mathrm{m} \times 93 \mu \mathrm{m}$. The grain colours represent locations in the inverse pole figure: [001] oriented grains are red, [101] green, and [111] blue (as a legend is available in Fig. 9 ). 


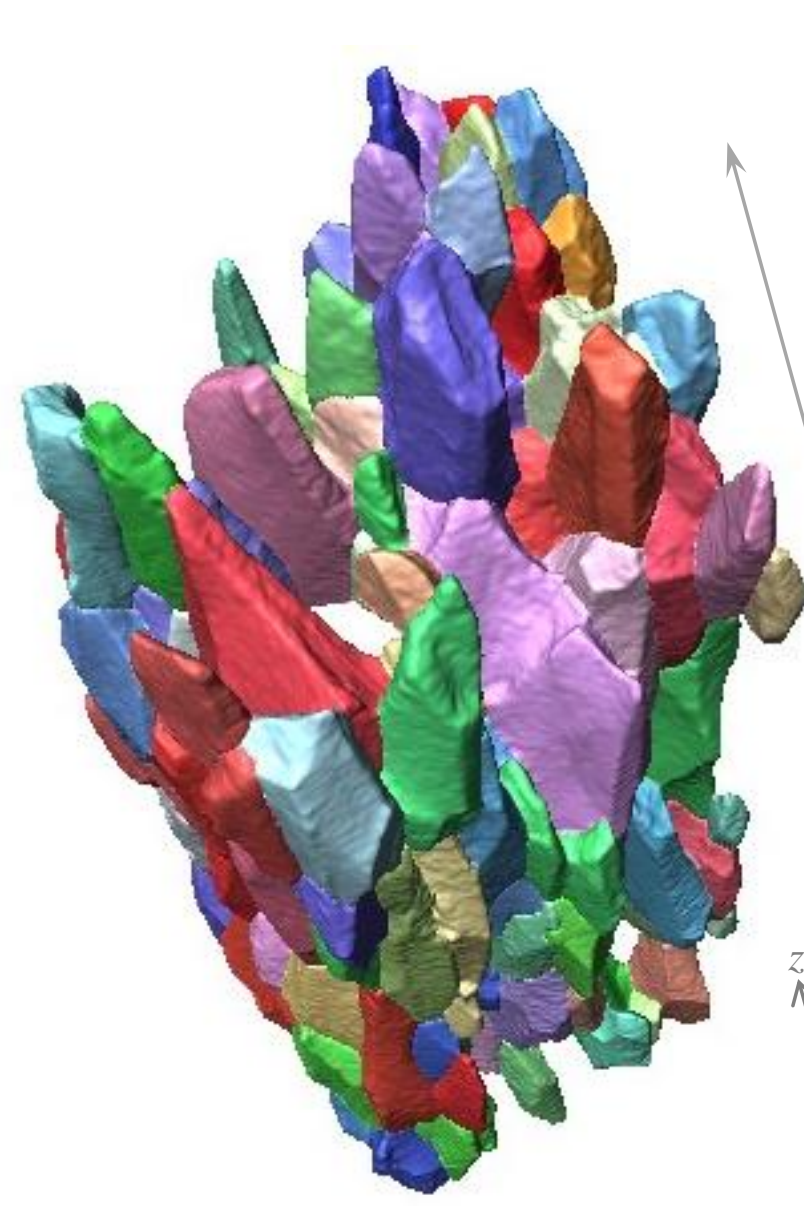

(a) 3D rendered image of grains before loading

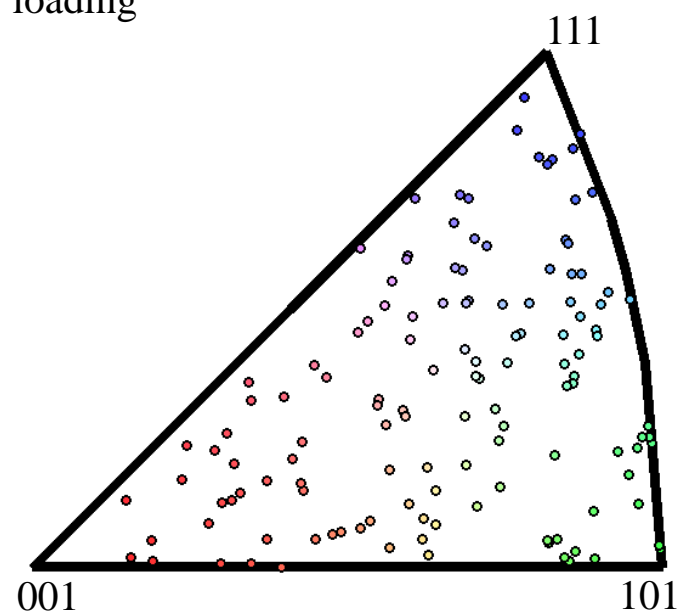

(c) Distribution of crystallographic orientations before loading

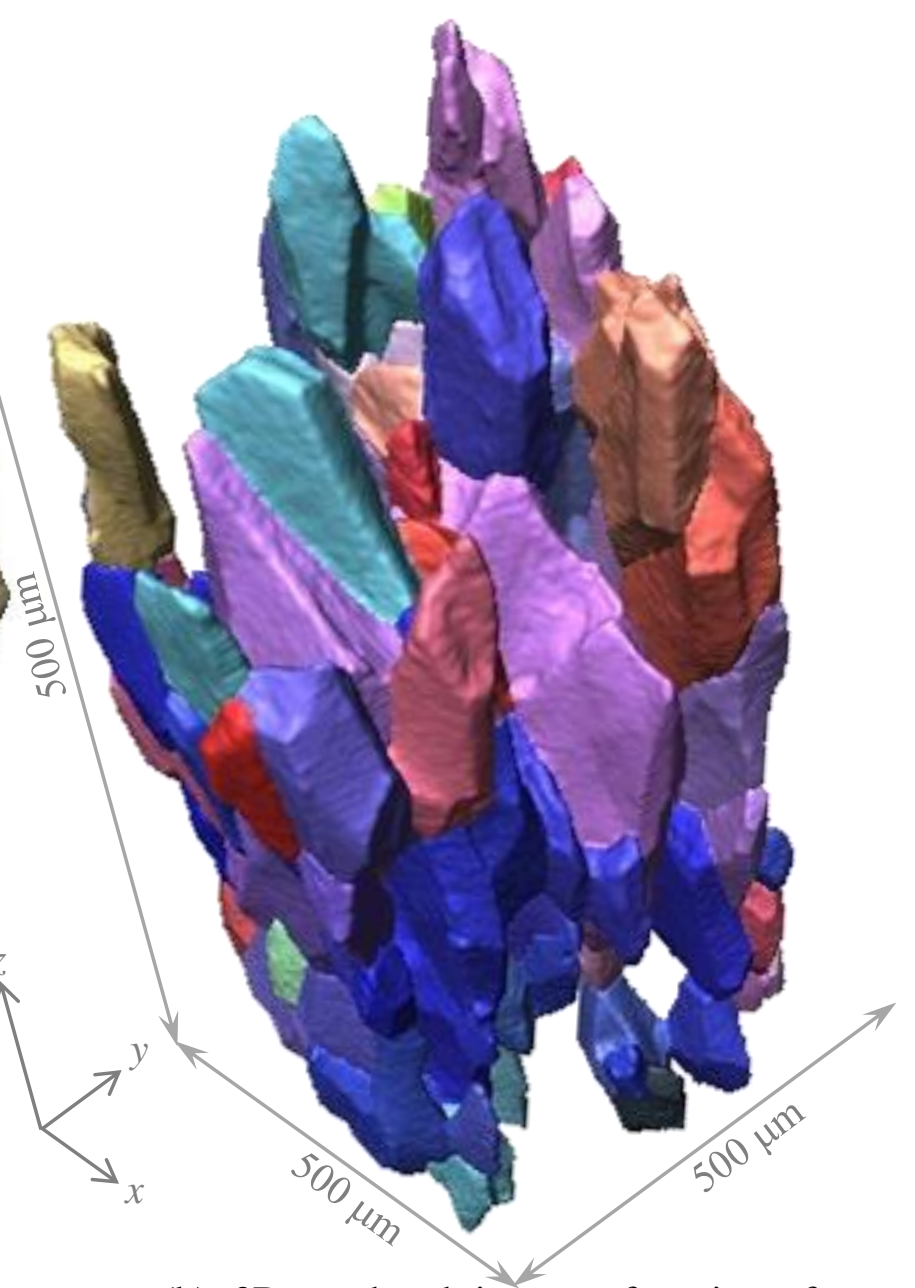

(b) 3D rendered image of grains after loading

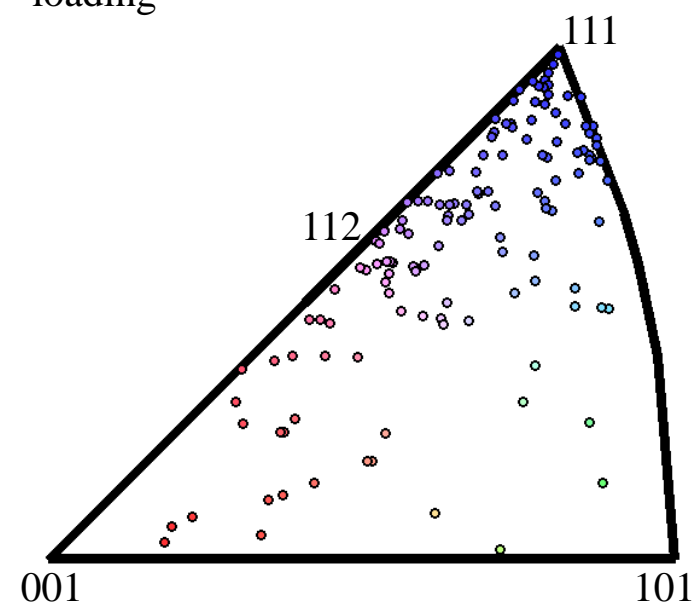

(d) Distribution of crystallographic orientations after loading

Fig. 9 Results of the DAGT analysis, representing the distributions of crystallographic orientations before and after loading of $27.0 \%$ in plastic strain. Note that strong tension texture is formed after deformation. The colours of grains and inverse pole markings represent locations in the inverse pole figures: [001] oriented grains are red, [101] green, and [111] blue. 


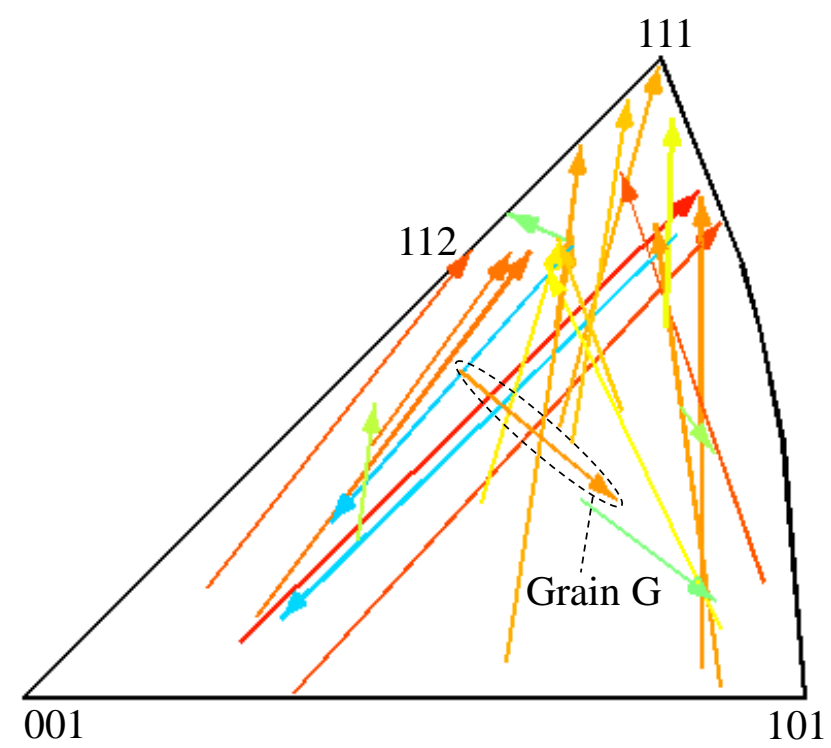

Fig. 10 Changes in orientation up to loading of $27.0 \%$ in plastic strain. The circled arrow shows the rotation of Grain $\mathrm{G}$ (highlighted in Fig. 11). 


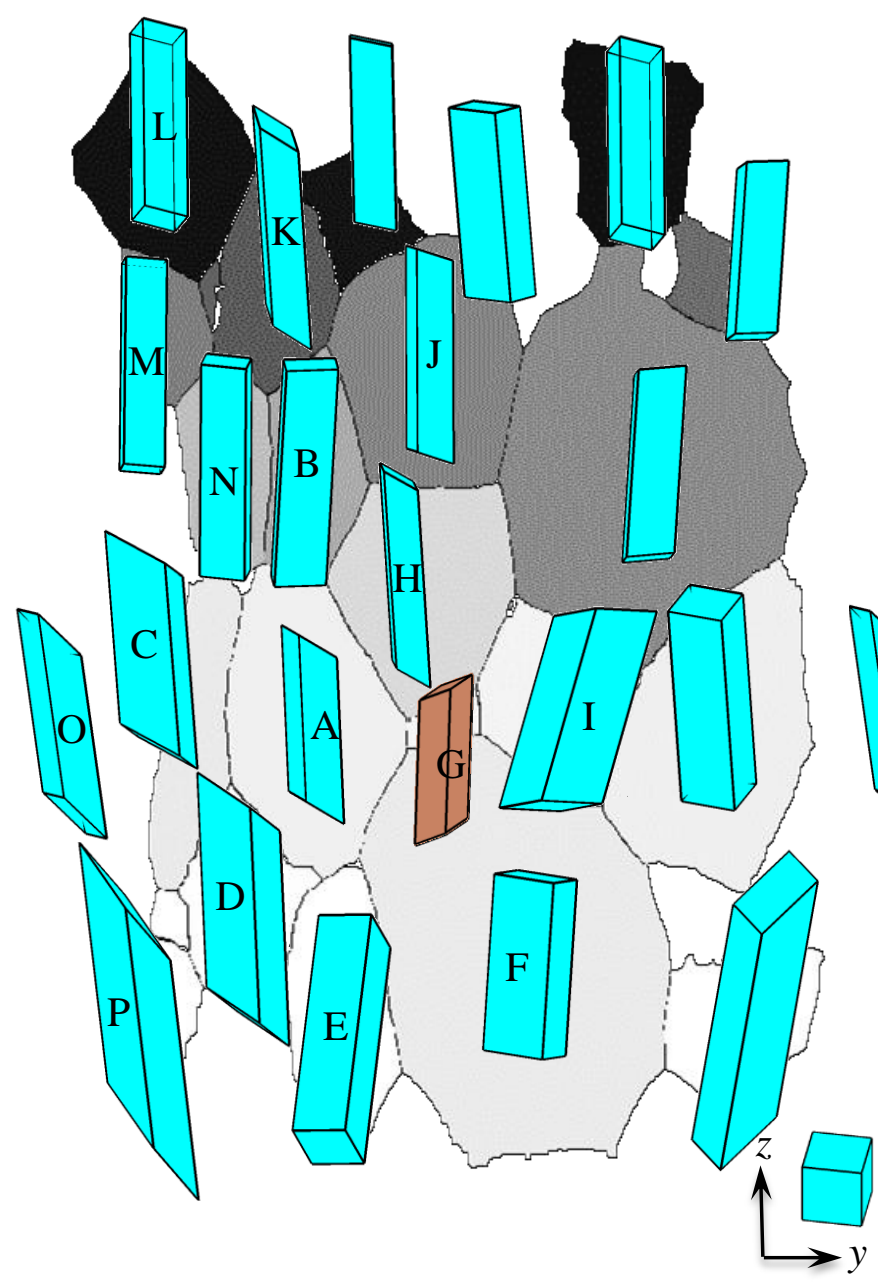

(a) Deformed unit box according to measured mean strain in each grain

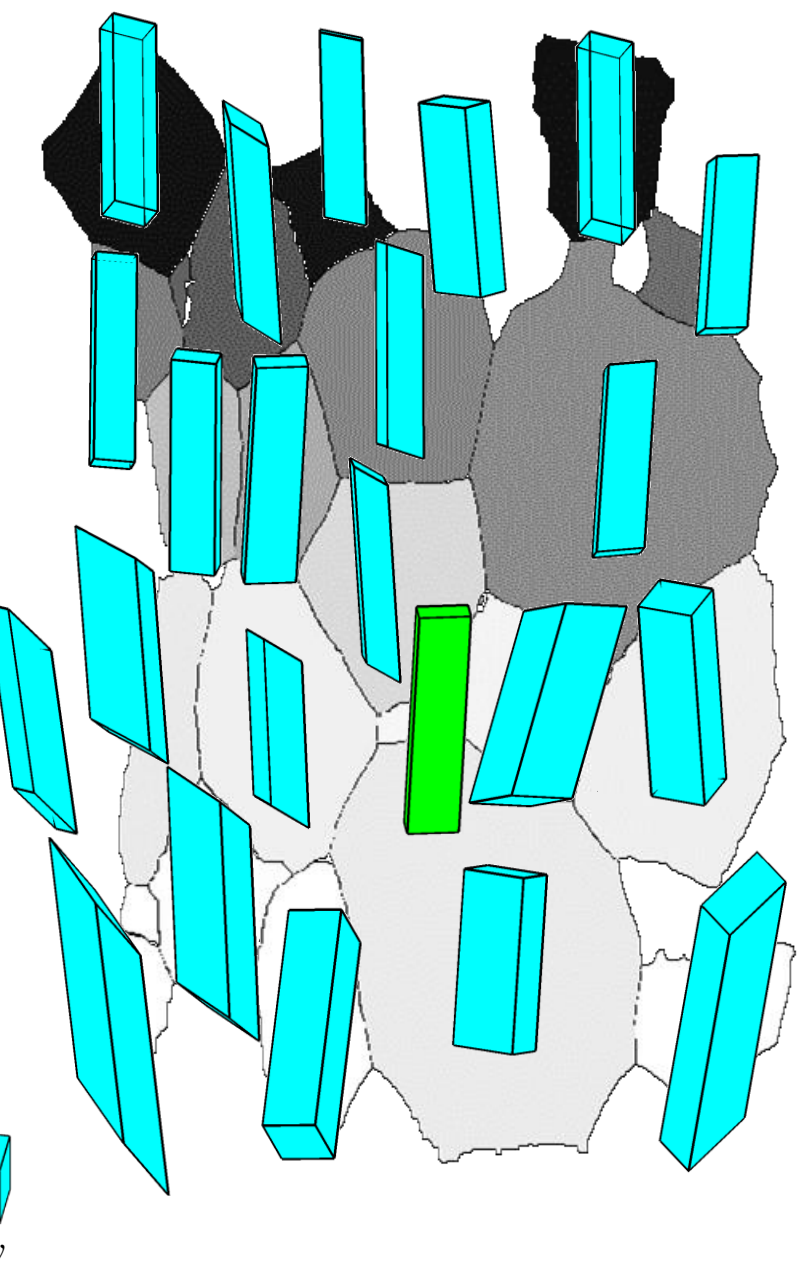

(b) Only the green box was replaced from (a) after recalculating under an assumption

Fig . 11 Representation of mean strain in individual grains, using unit boxes. A regular hexahedron located near the coordinate axis was deformed with the mean strain value of the respective grain, and positioned on a virtual cross-section, in (a). Note that strain values have been magnified five times to enhance visualisation. In (a), the deformed box highlighted in brown corresponds to Grain $\mathrm{G}$, which was rotated, during deformation, even farther away from the orientations of deformation texture shown in Figs. 9 and 10. In (b), only this box of interest (now highlighted in green) was virtually deformed, on the presumption that this grain was oriented to the nearest [111] direction. 

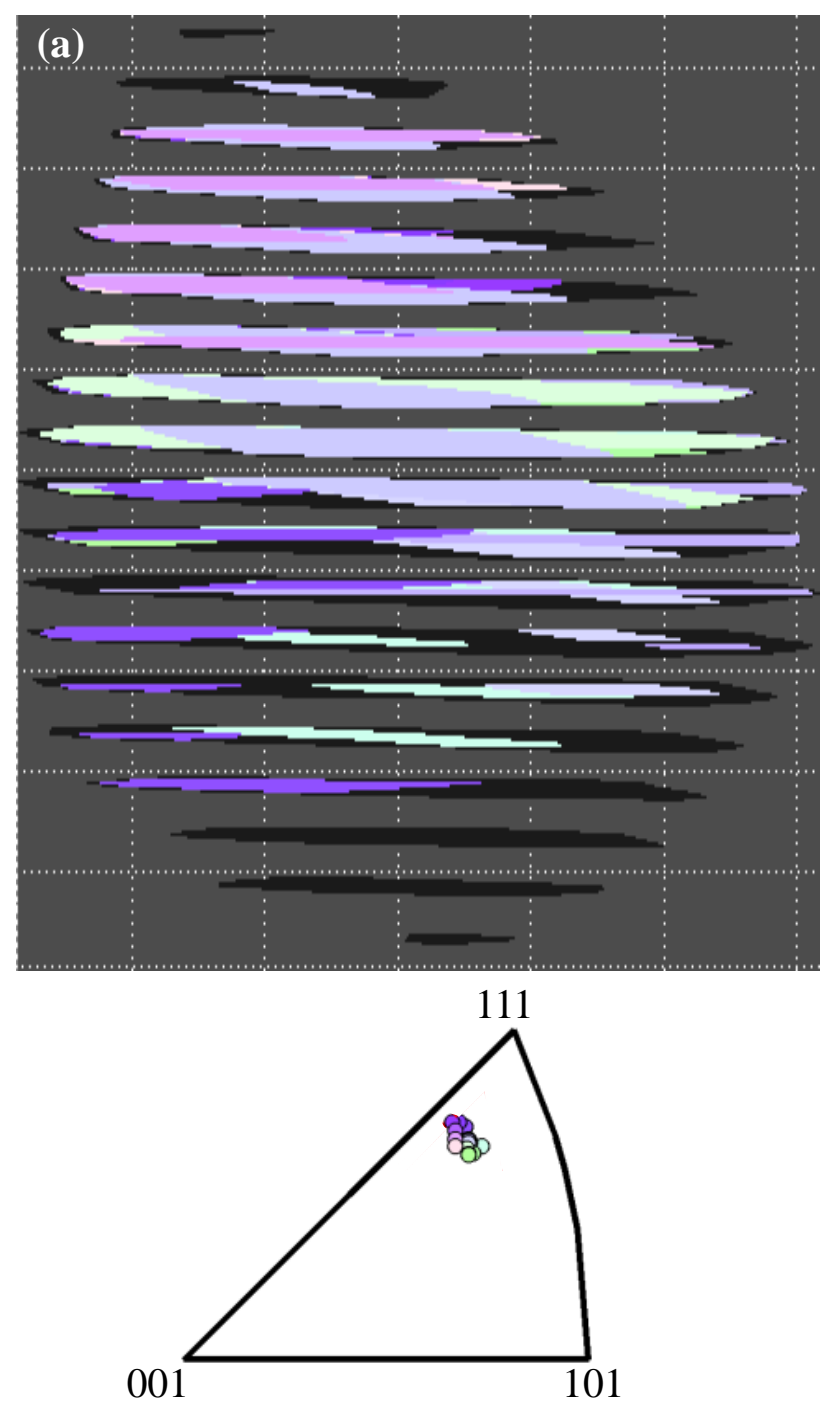
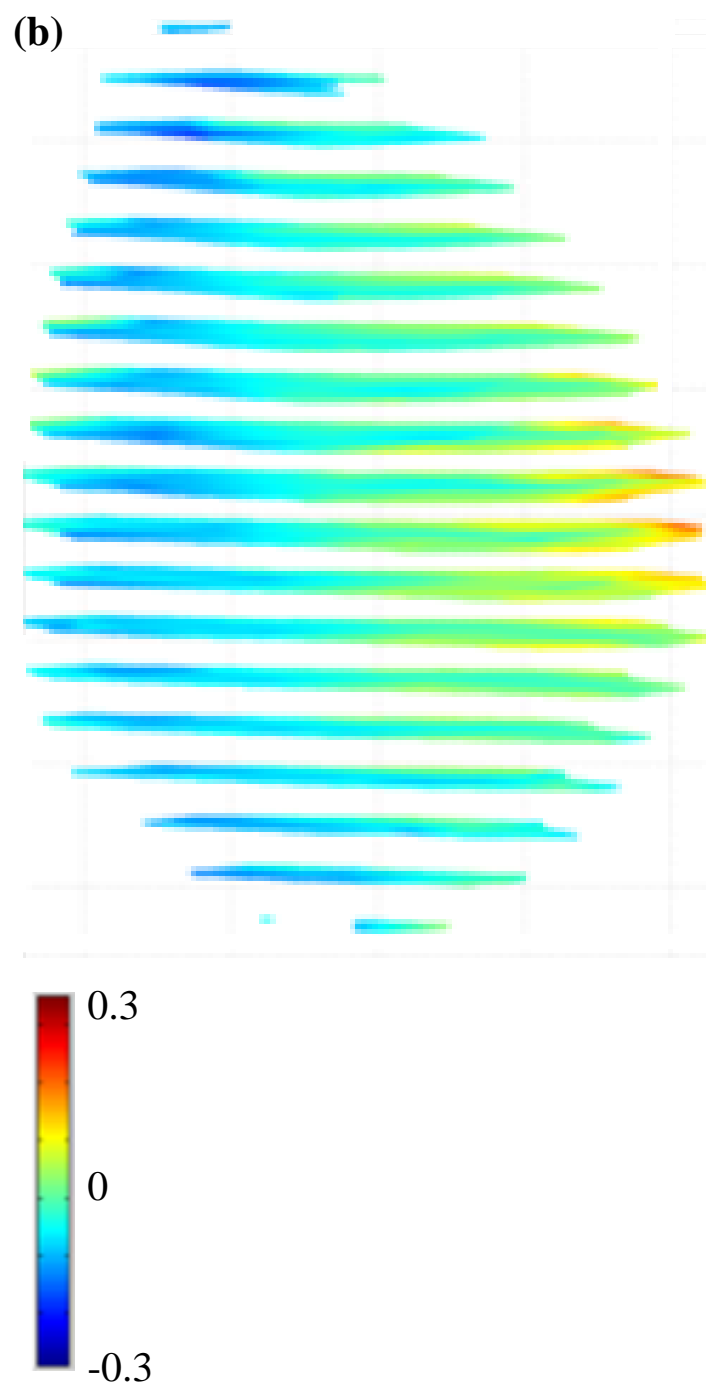

Fig. 12 Results of the DAGT analysis, representing the 3D distribution of crystallographic orientation in a grain (in (a)), and the corresponding 3D distribution of shear strain, $\gamma_{x y}$ (in (b)). The colours in (a) represent locations in the inverse pole figure. Note that the colour orientation is more enhanced in (a) than in Figs. 8 and 10, in order to express subtle differences in orientation. 

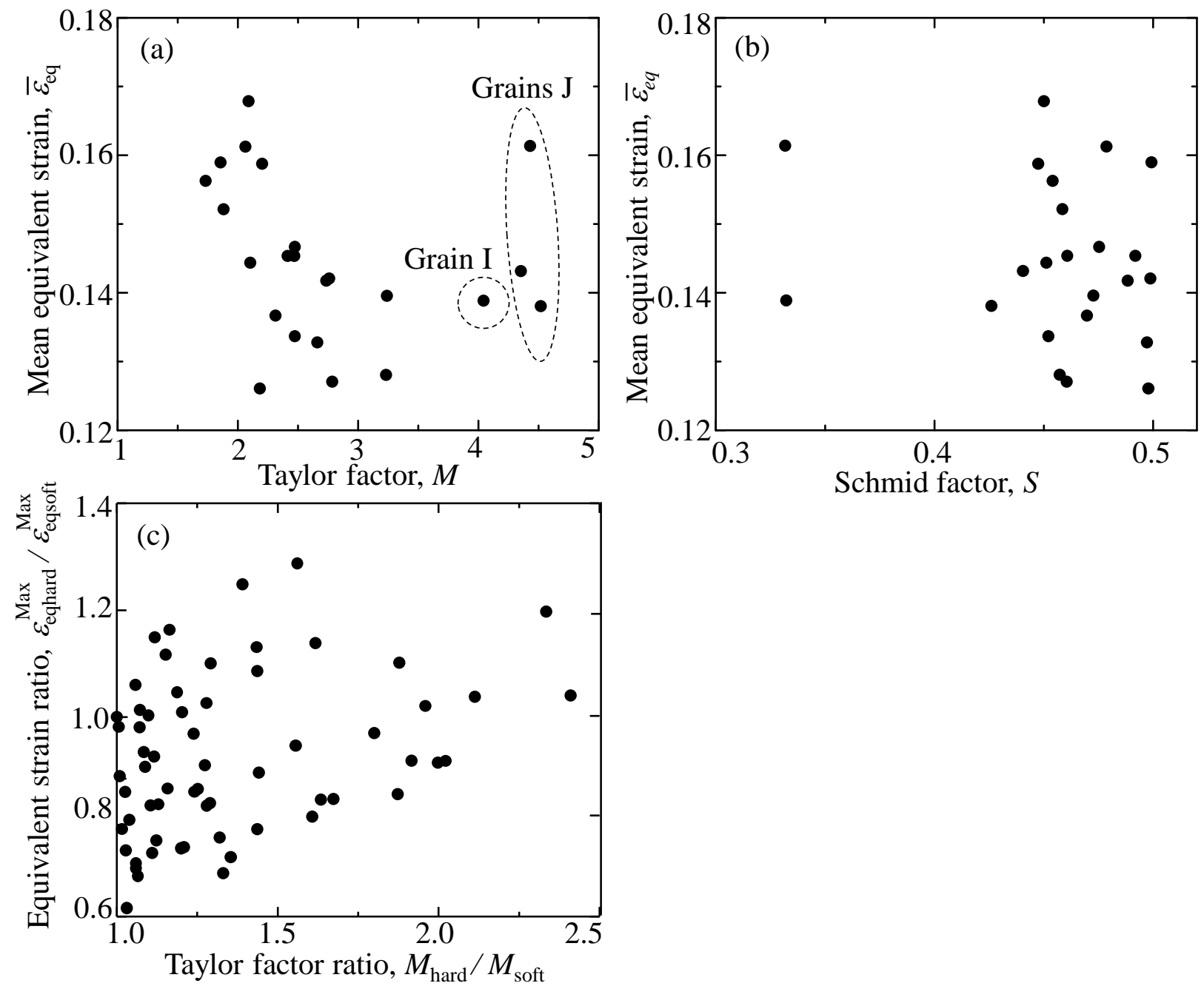

Fig. 13 Relationships between various crystallographic parameters and mean or maximum equivalent strain. In (c), the ratios of these parameters between neighbouring grains are plotted. 


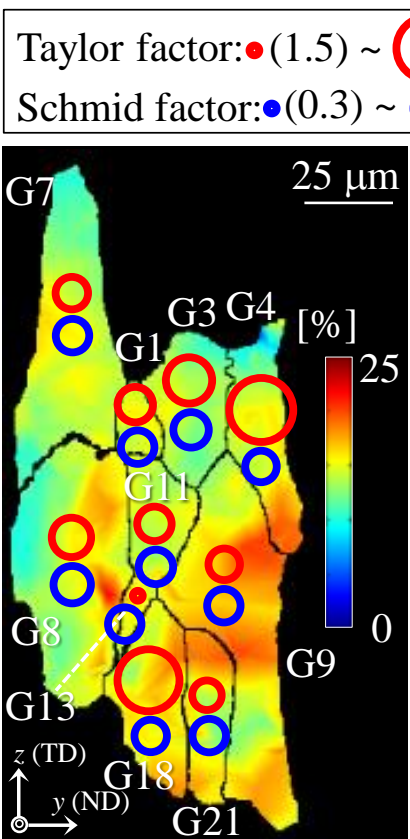

(b) $\varepsilon_{e q}$

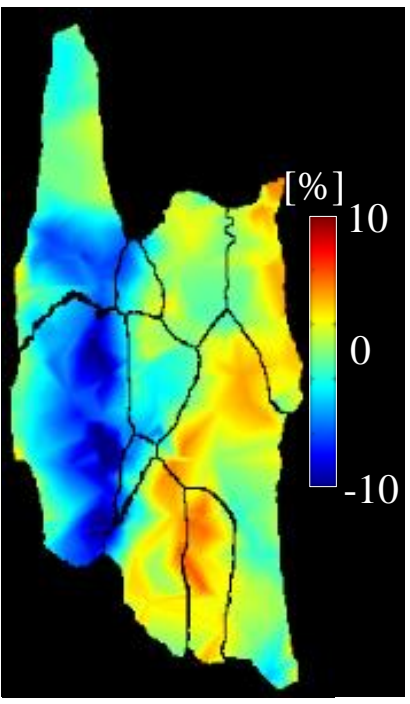

(f) $\gamma_{x y}$

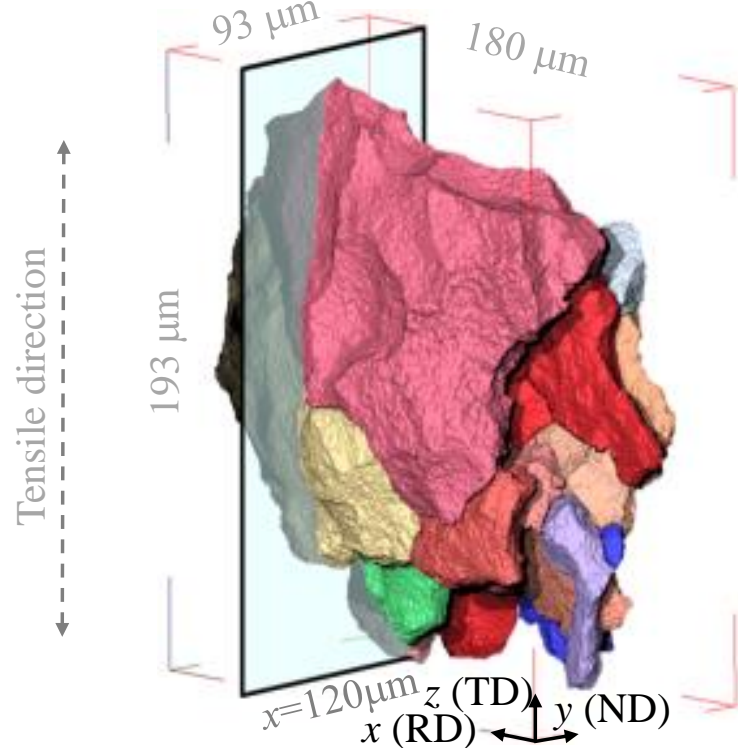

(0.5) (a) 3D extracted grains and the $y-z$ cross section used for (b) to (i)

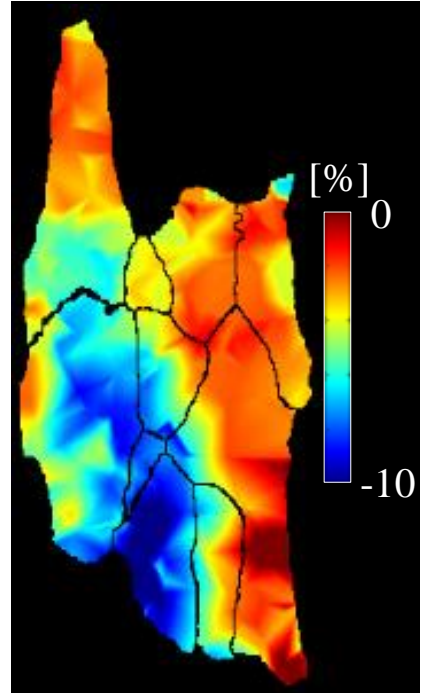

(c) $\varepsilon_{x x}$

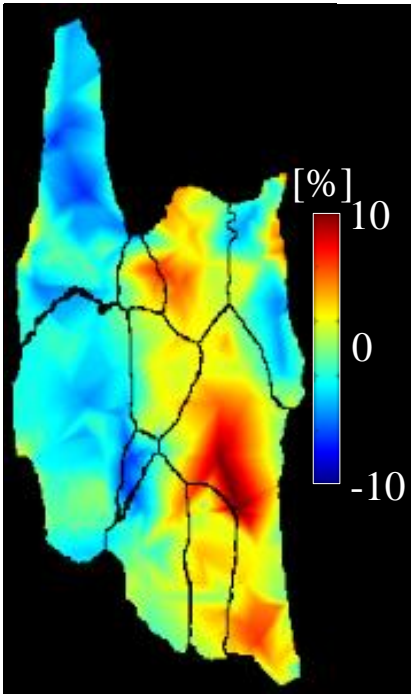

(g) $\gamma_{y z}$



(d) $\varepsilon_{y y}$

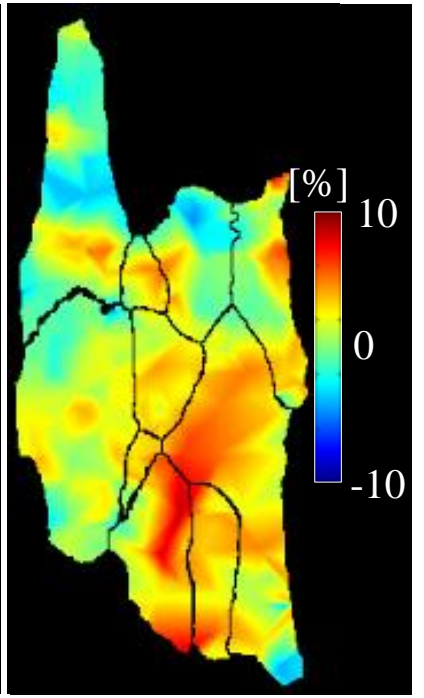

(h) $\gamma_{z x}$

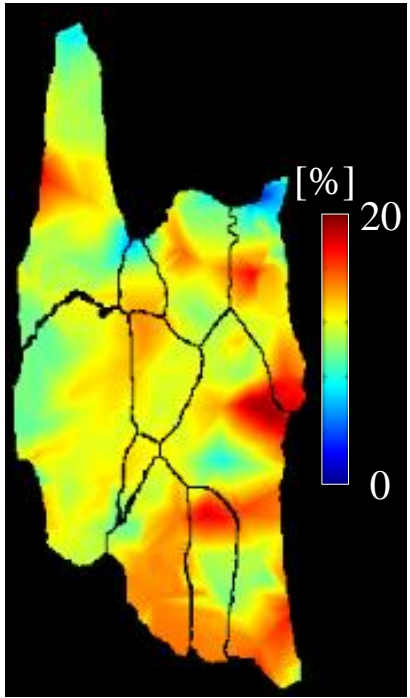

(e) $\varepsilon_{z z}$

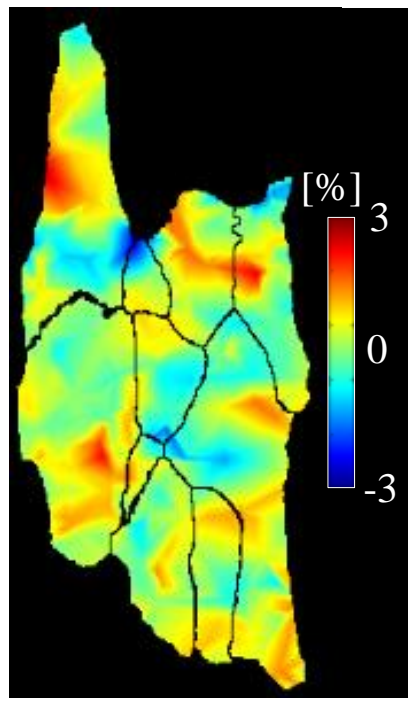

(i) $\varepsilon_{m}$

Fig . 14 Strain maps on a $y-z$ virtual cross-section (shown in (a)). Local plastic strain was calculated between applied strain, $\varepsilon_{\mathrm{a}}$, of 0 and $10.4 \%$. Plotted are: (b) equivalent strain, $\varepsilon_{\text {eq }}$; (c) normal strain in the $x$ (RD) direction, $\varepsilon_{x x}$; (d) normal strain in the $y$ (ND) direction, $\varepsilon_{y y}$; (e) normal strain in the $z$ (TD) direction, $\varepsilon_{z z}$; (f) shear strain in the $x-y$ direction, $\gamma_{x y}$; (g) shear strain in the $y-z$ direction, $\gamma_{y z}$; (h) shear strain in the $z-x$ direction, $\gamma_{z x}$; and (i) hydrostatic strain, $\varepsilon_{\mathrm{m}}$. Circle size in (b) denotes the magnitude of the local Taylor factor (red) and Schmid factor (blue). G1-G21 refer to the grains labelled in Fig. 8. 

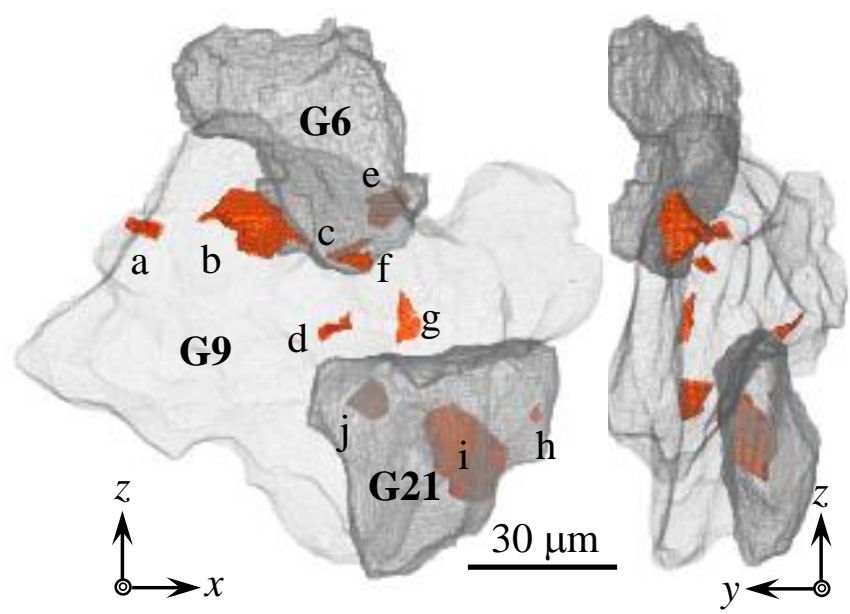

(a) View in the $y$ direction

(b) View in the $x$ direction

Fig. 15 3D representations of high-strain regions, A-I, in three grains (Grains 6, 9 and 21). Regions with $\varepsilon_{\mathrm{a}}$ greater than $20 \%$ are highlighted in red. 


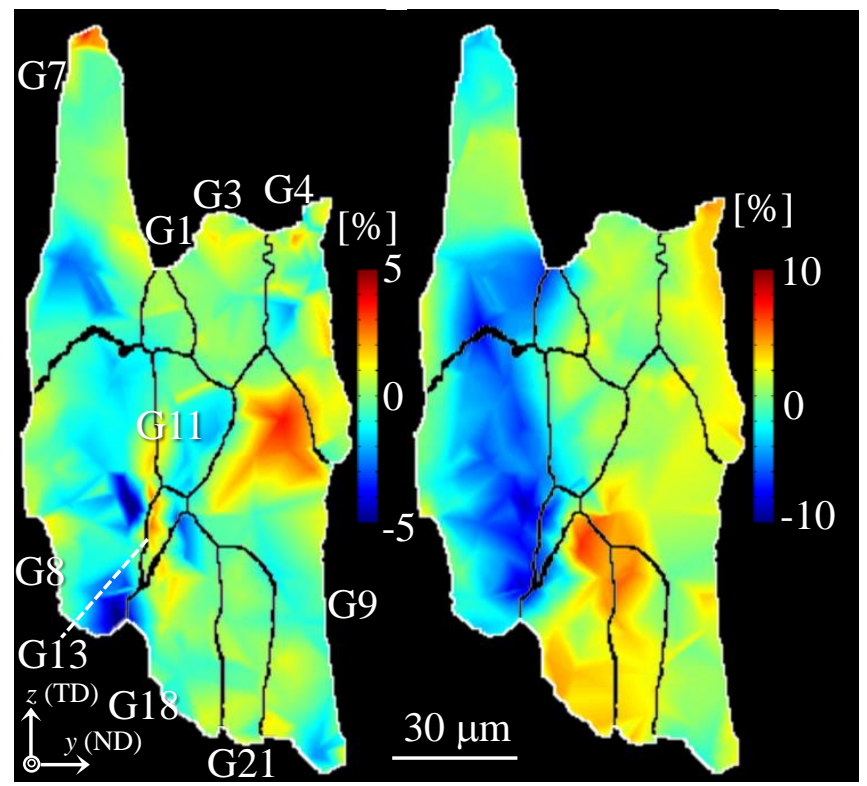
(a) $\varepsilon_{\mathrm{a}}=2.8 \%$
(b) $\varepsilon_{\mathrm{a}}=10.4 \%$
$\left(\Delta \varepsilon_{\mathrm{a}}=2.8 \%\right)$
$\left(\Delta \varepsilon_{\mathrm{a}}=7.6 \%\right)$

Fig. 16 Change in local shear strain $\left(\gamma_{x y}\right)$ distribution, viewed on the same $y-z$ virtual cross-section as in Fig. 14. Local plastic strain was calculated between $\varepsilon_{\mathrm{a}}$ of 0 and $2.8 \%$ in (a), and 2.8 and $10.8 \%$ in (b). Note the difference in contour levels between (a) and (b). 


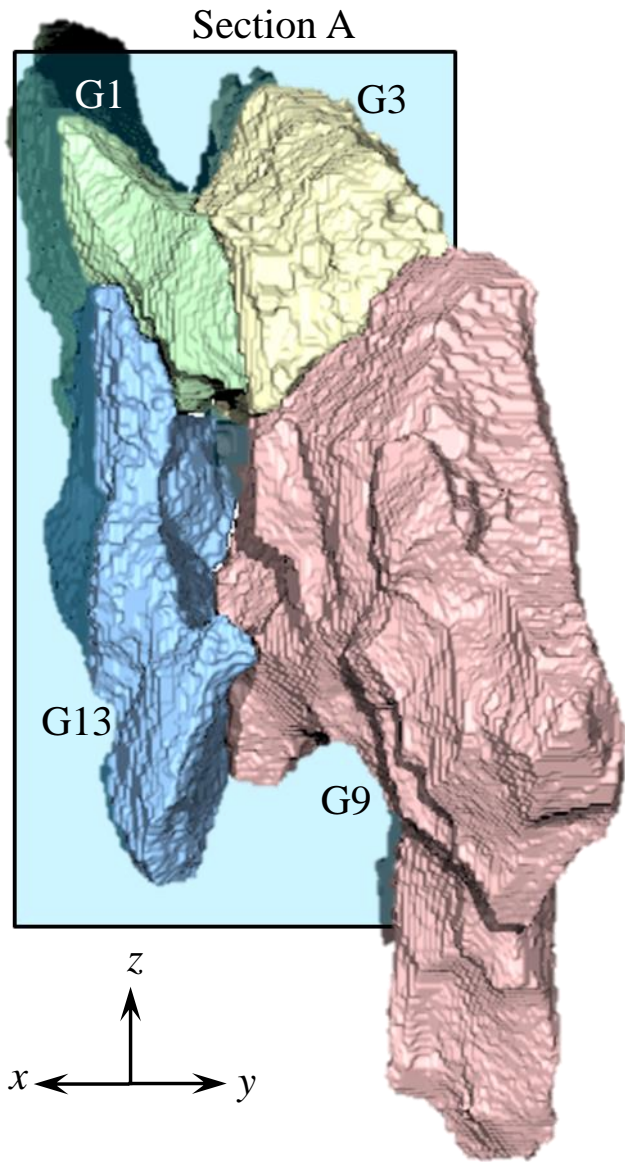

(a) Four grains located around a quadruple junction point
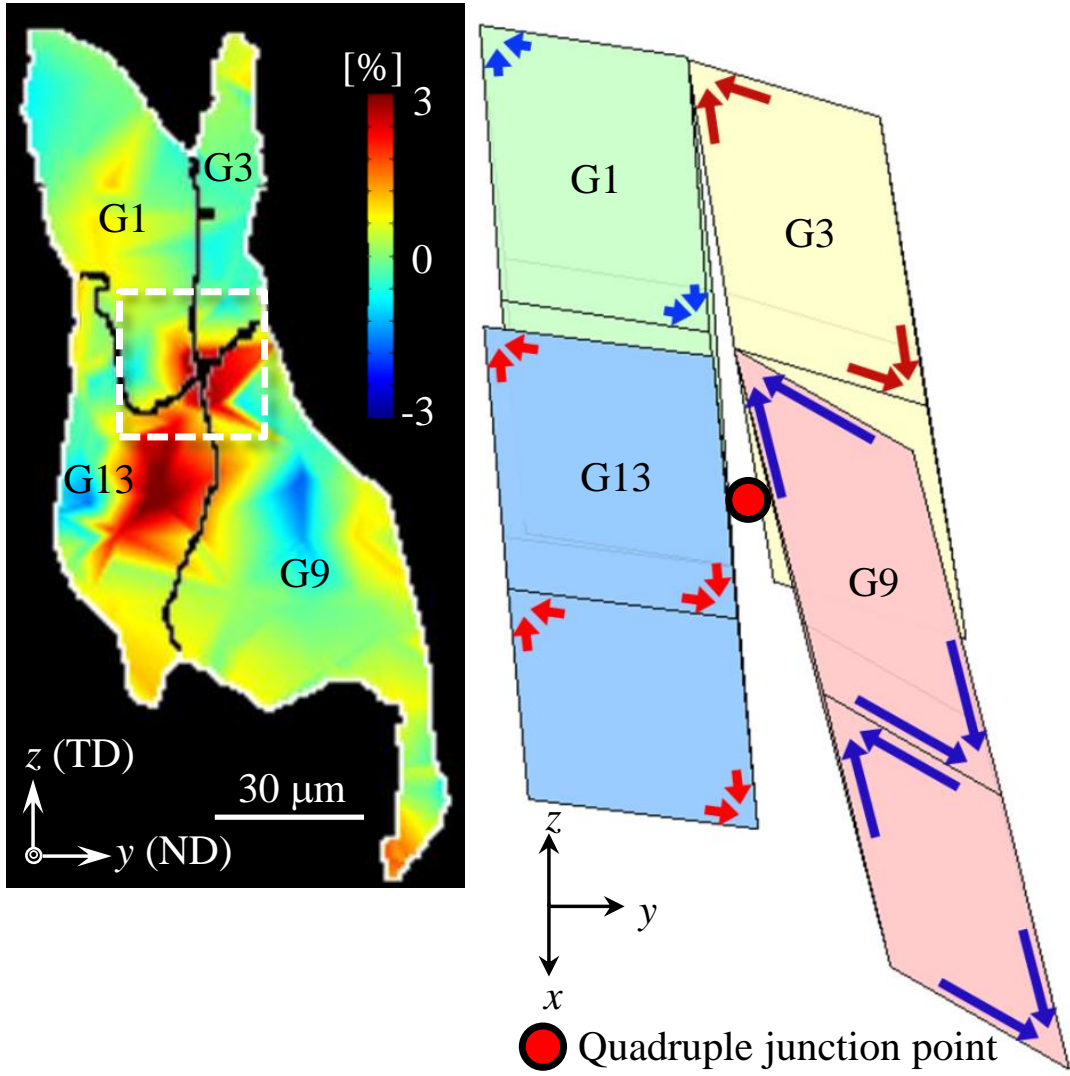

(c) Oblique view of the four deformed unit boxes (b) Hydrostatic strain map and a square region of interest around the quadruple junction point

Fig. 17 Representation of the mean strain in local regions of four neighbouring grains (defined by the dashed white box in (b)), with a unit box around the quadruple junction point. Local plastic strain was calculated between applied strain, $\varepsilon_{a}$, of 0 and $10.4 \%$. A regular hexahedron was deformed with the mean strain value of the specified region in each grain, as was demonstrated in Fig. 12. Note that the strain values have been magnified five times to enhance visualisation. The four grains in (a) are also shown in Figs. 14 and 15, and the hydrostatic strain distribution in Section A of (a) is shown in (b). 